\title{
Guiding and motivating students through open social student modeling: lessons learned
}

\author{
${ }^{1}$ I-Han Hsiao, ${ }^{2}$ Peter Brusilovsky \\ ${ }^{1}$ School of Computing, Informatics and Decision Systems Engineering, Arizona State \\ University, 699 S. Mill Avenue, Tempe, AZ, 85281, USA \\ ${ }^{2}$ School of Information Sciences, University of Pittsburgh, 135 N. Bellefield Ave, \\ Pittsburgh, PA, 15260, USA
}

\section{STRUCTURED ABSTRACT}

\section{Background/Context:}

A large number of educational resources are now made available on the Web to support both regular classroom learning and online learning. The abundance of available content has produced at least two problems: how to help students find the most appropriate resources and how to engage them into using these resources and benefit from them. Personalized and social learning have been suggested as potential ways to address these problems. Our work attempts to integrate these directions of research by combining ideas of adaptive navigation support and open student modeling with the ideas of social comparison and social visualization. We call our approach Open Social Student Modeling (OSSM).

\section{Objective/Research Questions:}

In this paper, we are trying to achieve two goals. First, we review a sequence of our earlier projects focused on Open Social Student Modeling for one kind of learning content and formulate several key design principles that contribute to the success of OSSM. Second, we present our exploration of OSSM in a more challenging context of modeling student progress for two kinds of learning content in parallel. This part aims to answer the following research questions: How do we design OSSM interfaces to support many kinds of learning content in parallel? Will current identified design principles (key features) confirm the power of the learning community through OSSM with multiple learning resource collections? Will the OSSM visualization provide successful personalized guidance within a richer collection of educational resources?

\section{Research Design:}

Four classroom studies were designed to assess the value of different designs options for OSSM visualization for one and multiple kinds of learning content in the context of programming language learning. The authors examine comparative success of different design options to distill successful design patterns and other important lessons for the future developers of OSSM for personalized \& social e-learning.

\section{Findings/Results:}

The results confirmed that the motivational impact of personalized social guidance provided by OSSM system in the target context. The interface encouraged students to explore more topics and motivated them to do some work ahead of the course schedule. Both strong and weak students worked with the appropriate levels of questions for their 
readiness, which yielded a consistent performance across different levels of complex problems. Additionally, providing more realistic content collection on the navigation supported open social student modeling visualizations resulted in uniform performance for the group.

\section{Conclusions/Recommendation:}

A sequence of studies of several Open Social Student Modeling interfaces confirmed that a combination of adaptive navigational support, open student modeling, and social visualization in the form of OSSM interface, could reinforce the navigational and motivational values of these approaches. In several contexts, OSSM interface demonstrated its ability to offer effective guidance helping students to locate the most relevant content at the right time while increasing student motivation to work with diverse learning content. 


\section{Executive Summary}

\section{Introduction}

The executive summary is organized into 4 sections: 1) the vision of the project, 2) description of the learning context, 3) educational technology - open social student modeling, 4) significance in education and lessons learned about learning technology design for personal and social e-learning.

\section{The Vision of the Project}

A large number of educational resources are now available on the Web to support both regular classroom learning and online learning. This abundance of available content produces at least two problems: how to help students find the most appropriate resources and how to engage them in using these resources and benefiting from them. Personalized learning and social learning technologies, among others, have been explored to address these problems. Personalized learning focuses mostly on guiding learners to good learning resources to help every learner find the most relevant and useful content given a learner's current state of knowledge and interests. Social learning, among other positive impacts on the educational process, is known for its ability to increase motivation of students to learn. While each of these technologies has been explored in many research projects, very few attempts have been made to use these technologies in combination.

We believe the integration of personalized and social learning technologies is a very promising research direction. These technologies have complementary strengths and could potentially reinforce each other when applied together. This paper reports the results of our exploration of one specific educational technology at the crossroads of personalized and social learning: Open Social Student Modeling.

\section{Description of the Learning Context}

In programming language learning, one usually learns by performing multiple kinds of activities and interacting with multiple types of learning content, i.e., reading textbooks, exploring program examples, writing programs, watching video lectures etc. In this work, we examined the value of open social student modeling as an interface to access one and two types of learning content: problem-solving quizzes and annotated examples. The content is offered online as non-compulsory learning resources for students to study, practice, and self assess their knowledge.

\section{Educational Technology - Open Social Student Modeling}

Open Social Student Modeling (OSSM) integrates adaptive navigation support and open student modeling, two prominent educational technologies in the field of personalized learning and social learning. OSSM can be considered a social extension of open student modeling, a technology to externalize student models that provides adaptation effects in an adaptive educational system. In this work, we review several iterations of OSSM design and classroom studies for single-content access, formulate key design principles for a successful OSSM educational system, and confirm these principles in a more complex OSSM interface for two types of learning content. The list of principles includes:

- Content access: Direct access to the learning content through Open Social Student Modeling interfaces is important for leveraging the value of OSSM. While classic open models offer no links to access content, our studies shows examining own or peer models students frequently discover lack of knowledge on a specific topic and want to act immediately by working with related content. To support this workflow, 
access to content should be provided from both personal and peer knowledge visualization.

- Sequence: OSSM should leverage the natural sequence of course topics. A sequential content organization aligned with course topics, provides efficient learning guidance and allows students to interpret their progress in the course context

- Identity: Comparing student feedback in QuizMap with its fragmented presentation of personal knowledge and Progressor with a clear concentrated representation of personal knowledge, we found the importance of identity in knowledge visualization. A successful visualization of personal knowledge should capture all information related to the target student and display it in a clear form. It allows students to identify themselves with the OSSM and easily compare their state of knowledge with each other.

- Peer comparison: Enabling peer-level comparisons of students' learning progress is important. It increases student motivation to work and achieve better performance. Peer comparison implies exposing student models not only to the target learner, but also to peers and provide an interface for its exploration. Our findings reveal that students view the openness of the personal model to peers positively.

- Guidance: The organization of OSSM visualization should support personal and social guidance, i.e., helping students to identify most critical lack of knowledge by comparing their progress with class or peers and most relevant direction to expand knowledge given the current state of the course by following the crowd.

\section{Significance in Education and Lessons Learned}

- This work reviews and reports the value of OSSM, an approach that combines personalized and social learning technologies. It expands earlier work on OSSM by comparing several OSSM designs for single type of learning content and examining it in a more challenging context with two kinds of content. Altogether, the presented research confirms the navigational and motivational values of OSSM. Our results demonstrate the OSSM increases student motivation to work with practice learning content and increases problem-solving success in the domain of programming.

- Based on the sequence of OSSM studies, this work formulates key design principles for successful application of open social student modeling in the context of programming courses.

- $\quad$ This reported work suggests and explores a scalable approach to offer OSSM for realistic educational context where students are expected to work with several types of learning content.

- This reported work suggests and explores a scalable approach to offer OSSM for realistic educational context where students are expected to work with several types of learning content. 


\section{Guiding and motivating students through open social student modeling: lessons learned}

\section{Introduction}

A large number of educational resources are now available on the Web to support both regular classroom learning and online learning. This abundance of available content produces at least two problems: how to help students find the most appropriate resources and how to engage them in using these resources and benefiting from them. Personalized learning and social learning technologies, among others, have been explored to address these problems. Personalized learning focuses mostly on guiding learners to good learning resources to help every learner find the most relevant and useful content given a learner's current state of knowledge and interests (Kay, 2008). Social learning, among other positive impacts on the educational process, is known for its ability to increase motivation of students to learn (Barolli, 2006; Méndez, 2006; Vassileva \& Sun, 2008). While each of these technologies has been explored in many research projects, very few attempts have been made to use these technologies in combination. We believe, however, that the integration of personalized and social learning technologies is a very promising research direction. These technologies have complementary strengths and could potentially reinforce each other when applied together. This paper reports the results of our exploration of one specific technology at the crossroads of personalized and social learning: Open Social Student Modeling.

Open Social Student Modeling (OSSM) integrates adaptive navigation support (Brusilovsky, 2007) and open student modeling (Bull \& Kay, 2007), two prominent technologies in the field of personalized learning with social visualization, a popular approach in the field of social learning (Vassileva, 2008). OSSM can be considered a social extension of open student modeling. Open student modeling has been suggested as a way to externalize student models, the key component of any personalized learning systems. While in a traditional personalized learning system this model is usually hidden from the student and only used by the personalization engine to provide adaptation effects (Figure 1 left), systems with an open student model expose this model to the learner and provide an interface for its exploration and possible editing (Figure 1 right). Open student modeling is known for a number of positive effects. It increases the transparency of personalization, helps raise the students' awareness of their learning performances, and supports meta-cognitive processes (Bull \& Kay, 2013). In combination with adaptive navigation support, it can also efficiently guide students to the appropriate content QuizGuide (Brusilovsky, et al., 2004). In this context, the idea of Open Social Student Modeling is simply to make the content of individual and student models accessible not only to the target student herself, but to the a broader group of students, for example, students in the same class. The most natural way to do it is through social visualization that can visually present the content of multiple student models to the target student in a form that enables comparison of her own knowledge to the knowledge of her peers and the class as a whole.

We have explored the idea of OSSM in a sequence of studies. While the OSSM idea itself is relatively straightforward, it took us several attempts to "do it right" (i.e., implement it in a form that delivers several benefits) in a simple context with one type of learning content. We went through a sequence of incrementally more powerful designs that also allowed us to learn some important lessons about OSSM design. Armed with the lessons learned, we also approached a more challenging context and implemented OSSM visualization for two kinds of learning content in parallel. 
This paper presents an account of our work on OSSM over the last several years. We start with a literature review of open user modeling, social visualization, and underlying theories such as self-regulated learning and social comparison. Following that, we briefly summarize a sequence of our studies with OSSM in one-content-type context. These studies have been published before; we review them here to illustrate the problems of OSSM design and to present lessons learned from these studies. Next, we present in greater detail our more recent study that evaluated the OSSM interface for two types of content. At the end of the paper we summarize the results and discuss the limitations and consider future work.

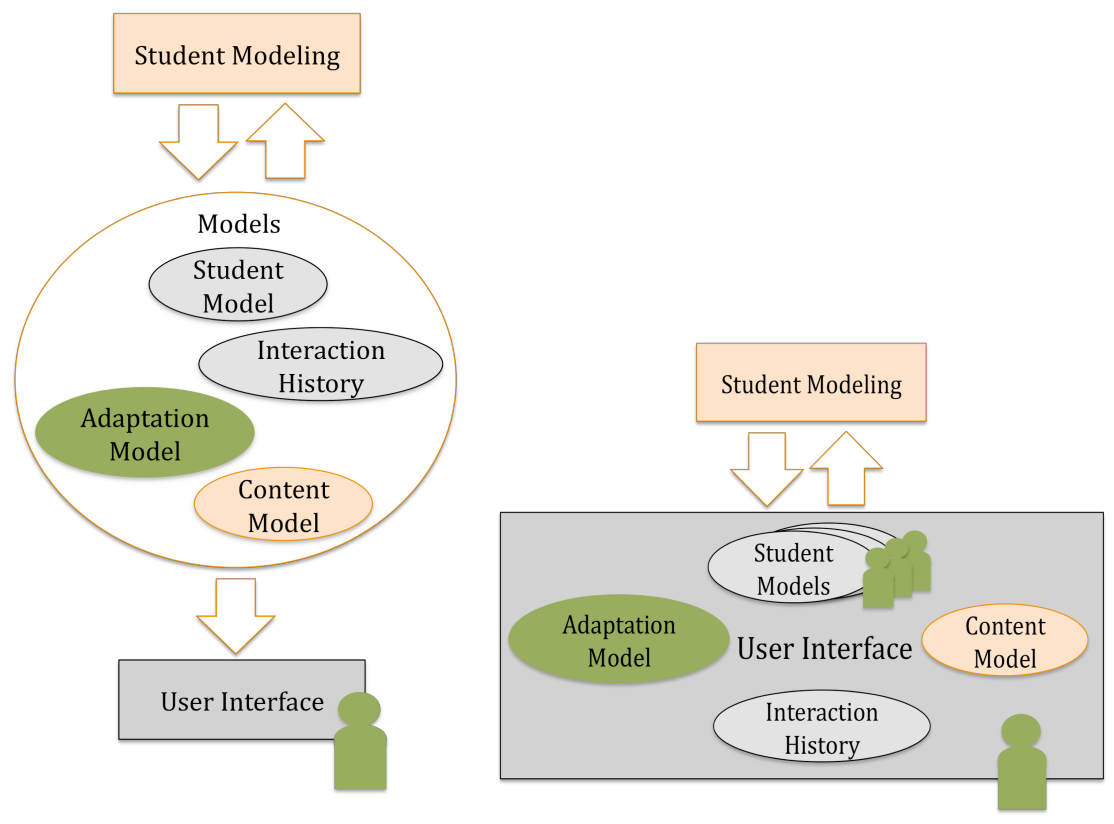

Figure 1. Traditional approach (left) vs. Integration of students' models into the interface (right)

\section{Background}

\subsection{Open Student Modeling}

An open student (learner) model is a special kind of student model that allows the student to access and possibly modify the model content. In traditional personalized learning systems, student models are hidden "under the hood" and used for the system's internal needs (i.e., to make the education process personalized) (Figure 1 left). The proponents of open student models (Figure 1 right) argue that the ability to view and modify their models could be beneficial for the students for a number of reasons. A typical open learner model displays the modeled state of student knowledge, although the examples of models displaying interests (Ahn, et al., 2007) or learning styles (Triantafillou, et al., 2004) are also known. Open knowledge models can be presented in simple forms such as a skill meter, a part-shaded bar showing learner progress as a subset of expert knowledge (Bull \& Kay, 2007; Weber \& Brusilovsky, 2001); the probability that a learner knows a concept (Corbett, 1995); or a user's knowledge level compared to the combined knowledge of other groups of users (Linton, et al., 2000). Skill meters have been extended to show progress as a subset of material covered which is, in turn, a subset of expert knowledge (Mitrovic \& Martin, 2007); or a further extension also allowing the existence of misconceptions and size of topic to be included in the skill meter (Bull \& Kay, 2007). 
There are two main streams of work on open student models. One stream focuses on the interfaces visualizing the model to support students' self-reflection and planning; the other one encourages students to participate in the modeling process, such as engaging students through the negotiation or collaboration on construction of the model.

Visual representations of the student model vary from displaying high-level summaries (such as skill meters) to complex concept maps or Bayesian networks. Corbett et al. (1995) described the ACT Programming Tutor interface that provides the learner with a skill meter showing the list of learning goals and the progress the learner has already made with respect to the goals. Mabbott and Bull (2004) elaborated on an interface providing students with four views over their learner models. These views visualize different aspects of the underlying domain knowledge model, namely the hierarchical structure of topics, lecture structure, semantic relationships among the topics, and the recommended sequence for learning the topics. The STyLE-OLM interface proposed by Dimitrova (2003) allows students to browse and navigate through their learner models using the visual notation of concept graphs.

Dimitrova et al. (2001) explored interactive open learner modeling by engaging learners to negotiate with the system during the modeling process. Chen et al. (2007) investigated active open learner models in order to motivate learners to improve their academic performance. Both individual and group open learner models were studied and demonstrated an increase of reflection and helpful interactions among teammates. Bull \& Kay (2007) described a framework to apply open user models in adaptive learning environments and provided many in-depth examples. Studies showed that students have a range of preferences for presentations on viewing their own knowledge in the open student modeling systems. Students highly value the options of having multiple views and being able to select one, which they are the most comfortable with. Such results are promising for potentially increasing the quality of reflection on their own knowledge (Mabbott \& Bull, 2004). A range of benefits have been reported on opening the student models to the learners, such as increasing the learner's awareness of the developing knowledge difficulties and the learning process, and students' engagement, motivation, and knowledge reflection (Bull, 2004; Mitrovic \& Britland, 2007; Zapata-Rivera \& Greer, 2000). In our own work on the QuizGuide system (Hsiao, et al., 2010) we embedded open learning models into adaptive link annotation and demonstrated that this arrangement can remarkably increase student motivation to work with non-mandatory educational content.

\subsection{Social Visualization and Social Navigation Support in E-learning}

Within a broader area of social learning, social navigation support and social visualizations are most directly related to the OSSM approach presented in the paper. Social navigation support captures a known social phenomenon by following the "footprints" of other people (Brusilovsky, et al., 2004; Dieberger, 1997, 2000; Wexelblat, 1999). The educational values have been confirmed in several studies (Brusilovsky, et al., 2009; Farzan \& Brusilovsky, 2008; Kurhila, et al., 2006). Social visualization aims to represent or organize multiple students' data in an informative way, for example, by producing visual representations of student groups. Group visualizations have been used to support the collaboration between learners among the same group, and to foster competition in a group of learners (Vassileva \& Sun, 2007). Vassileva and Sun (2007) investigated community visualization in online communities. They summarized that social visualization allows peer-recognition and provides students the opportunity to build trust in others and in the group. CourseVis (Mazza \& Dimitrova, 2007) was one of the pioneer systems providing graphical visualization of multiple groups of users to teachers and learners. It helped instructors to identify some common problems in distance learning. 
In our own work, we try to move beyond visual representations of learning analytics by moving from action visualization to knowledge visualization. We combined cognitive aspects of open student modeling with social and visual aspects of social visualization and social navigation support by allowing students to explore and interact each other's models as well as a cumulative model of the class. This idea was first explored by Bull \& Britland (2007), who used OLMlets to research the problem of facilitating group collaboration and competition. Their results showed that optionally releasing the models to their peers increases the discussion among students and encourages them to start working sooner. The Open Social Student Modeling approach presented in this paper moves these ideas further. A series of Open Social Student Modeling designs presented in the paper demonstrates several benefits that could be obtained by merging open student modeling, social visualization, and social navigation support.

\subsection{Theoretical Background: Self-Regulated Learning and Social Comparison}

Theory

The theoretical background for our work on open student modeling and social visualization is grounded in research on self-regulated learning and social comparison theory.

Research in self-regulated learning examines students' metacognitive strategies for planning, monitoring, and modifying their management and control of their effort on classroom academic tasks (Pintrich \& De Groot, 1990). Self-regulated learning involves self-monitoring to optimally interpret feedback from their academic learning (Zimmerman, 1990). Azevedo, et al. (2004) investigated how self-regulated learning helped students acquire conceptual understanding. The results showed that students who gained higher conceptual understandings (AKA: high jumpers) tended to be good at regulating their learning by using effective strategies, planning their learning by creating sub-goals and activating prior knowledge, monitoring their emerging understanding, and planning their time and effort. On the other hand, students who gained lower conceptual understandings (AKA: low jumpers) tended to handle task difficulties and demands by engaging mainly in help-seeking behavior, and did not spend much time monitoring their learning. Our work aims to leverage awareness, motivation, and content organization through social visualizations in the hopes of promoting students' self-regulated learning behavior.

Research in social comparison (Festinger, 1954) has demonstrated that people often determine appropriate behavior for themselves by examining the behavior of others, especially similar others (Buunk \& Gibbons, 2007). Consequently, it has been shown that individuals tend to behave similarly to their friends and peers (Cialdini, et al., 1999). Researchers and designers of online systems have used the insights from social comparison research in the study of online social behavior. In the educational domain, social comparison processes have been studied extensively (Darnon, et al., 2010; Kaplan $\&$ Maehr, 2007) and the positive impact on student performance has been examined in several papers (Light, et al., 2000; Huguet, et al., 2001). In online education environments, social comparisons were explored more recently (Vassileva, 2008), but no research to date has explored how social comparison-based adaptive systems can influence learning. Furthermore, while ample evidence points to the role of key personal attributes such as personality and culture in learning, little is known about how they impact learning in the context of adaptive learning systems or in environments in which social comparison is embedded. A synthesis review of many social comparison studies concluded that the upward comparisons in the classroom often lead to better performances (Dijkstra, et al., 2008). Over fifty years of social comparison theory literature, most of the research was done through qualitative studies using interviews, 
questionnaires and observation. In our research, we develop a set of quantitative measures for applying social comparison theory in the target context.

\section{Open Social Student Modeling: Studies with One Kind of \\ Learning Content}

Our work on Open Social Student Modeling was motivated by the success of our two earlier projects, QuizGuide (Brusilovsky, et al., 2004) and Knowledge Sea II (Brusilovsky, et al., 2004). The QuizGuide system applied open student modeling adaptive navigation support technology to provide personalized access to a collection of programming problems. The Knowledge Sea II system uses social navigation support and map-based visualization to help students navigate to most relevant weekly readings. QuizGuide demonstrated excellent ability to improve student performance and engage students to work with non-mandatory content. Yet its knowledge-based guidance was relatively hard to build; it required considerable knowledge engineering efforts to create a prerequisite-based network of topics. On the other hand, Knowledge Sea II used no knowledge engineering and its power to guide students to relevant reading was based on "social wisdom" - processing traces of student work. OSSM was born as an attempt to replace QuizGuide's original guidance approach based on labor- intensive knowledge engineering with self-organized social navigation that worked so well in Knowledge Sea II. We expected that by showing the state of the target student knowledge model, topic by topic, alongside student models of her peers will help the student to avoid topics that might be too easy or too hard to explore and focus on the most appropriate subset of programming problems. We also hoped that the engaging power of the open student model and the social comparisons provided by OSSM would work together in motivating students to do more work with non- mandatory learning content.

As it appears, the main challenge of our work on OSSM was to find the right visualization approach that could present students' own knowledge alongside peer knowledge in a form that promotes guidance and comparison. We had to go through three design iterations to build an OSSM interface able fulfill this promise. For each design iteration, we conducted at least one semester-long classroom study to evaluate the impact of OSSM on system usage and learning. Since the original idea of OSSM was to replace QuizGuide's knowledge-based guidance, all interfaces were designed to provide access to the same set of programming problems. As a result, we were able to use a Java version of QuizGuide (JavaGuide) as a baseline. The analysis of students' logs of their subjective opinions provided through questionnaires helped us to identify problems, learn important lessons, and informed the next design iteration. The following subsections describe the design rationales, setup and findings of three progressively improved OSSM interfaces.

\subsection{Exploring Open Social Student Modeling with QuizMap: adaptive navigation support of parameterized questions with TreeMap}

Our first attempt to design the OSSM interface was relatively close to the cell-based social navigation implementation in Knowledge Sea II. It used a space-filling TreeMap visualization approach (Shneiderman, 2004) and was promptly called QuizMap. The TreeMap approach was selected for its ability to efficiently represent hierarchical information and the map was built to represent three levels of hierarchy: (1) domain topics, (2) problems within each topic, and (3) the performance of individual students for each problem. Individual student performance for each problem was shown on the bottom level of the Treemap as a rectangle. The size of each rectangle (tile) represented the amount of work done by a specific student while the color indicated the amount of 
knowledge gained (increased with each successful answer). A student's own performance and the peer student performance were presented in contrasting colors (orange and blue accordingly). The exact values of problem attempts and knowledge gain were available as mouseovers by hovering over each tile. Based on the contrast of sizes and colors, one can estimate his/her current knowledge, relative standing in the group, and relative amount of effort to catch up to more capable peers. The map also allowed students to distinguish between new and hard topics (little class work, little success) and older, relatively learned topics (a lot of work and success). The QuizMap interface is presented in Figure 2. Note that to support exploration of new topic that had very little activity and small cells (upper right corner) it was possible to zoom in on a specific topic or question.

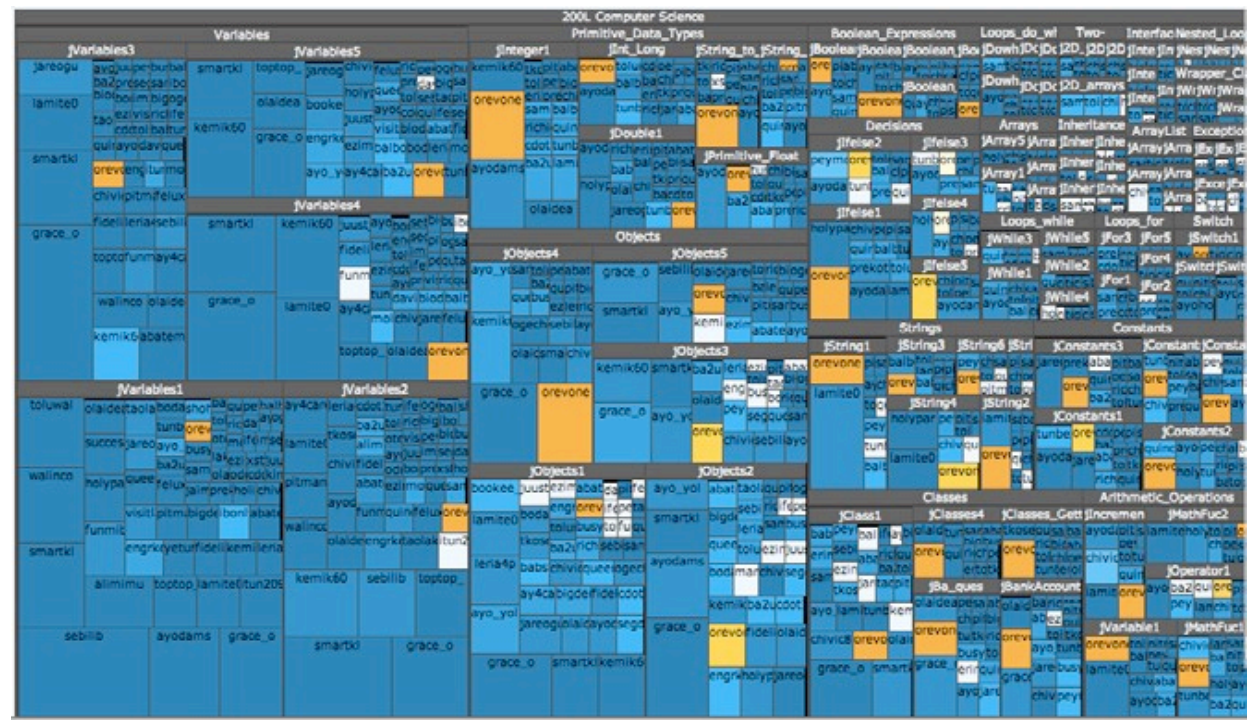

Figure 2. QuizMap interface.

An important design decision inherited from QuizGuide was the use of the map not just to visualize knowledge, but also to provide direct access to learning content. Following a mouse click in the area of a specific question, QuizMap presented a pop-up window to display the question. The questions were provided by the QuizJET system (Hsiao, et al., 2010) and were the same as in the earlier QuizGuide. Each question asks the student to predict the results of execution of a specific Java program (i.e., mentally execute the program and enter the final value of some variable of the text to be printed by the program.) All questions are parameterized, i.e., include a random parameter, which the system instantiates when the question is delivered to a student. As a result, the student can attempt to answer the same question multiple times with different values of the parameter, which helps to achieve the mastery level. The implementation and functionalities of parameterized self-assessment quizzes were described in detail in (Hsiao, et al., 2010).

Figure 3 left \& right show two different zoom-in scenarios of students' views. Figure 3-left demonstrated that student A had consistent performance in terms of attempts and success rate across all questions (jObject1 jObject5) in topic Object. Each cell grid size and color density shade is relatively similar. On the other hand, Figure 3-right showed that student $\mathrm{B}$ focused on working on certain questions at the expense of others. Considerable efforts were spent on jObject 4 question, which reached relatively high attempts. She also had a considerable amount of work done in questions jObject2 and jObject3 but achieved a lower success rate (lighter orange color). With QuizMap, students were expected to identify the strengths and weaknesses of themselves and their peers and estimate their readiness for each available question. 

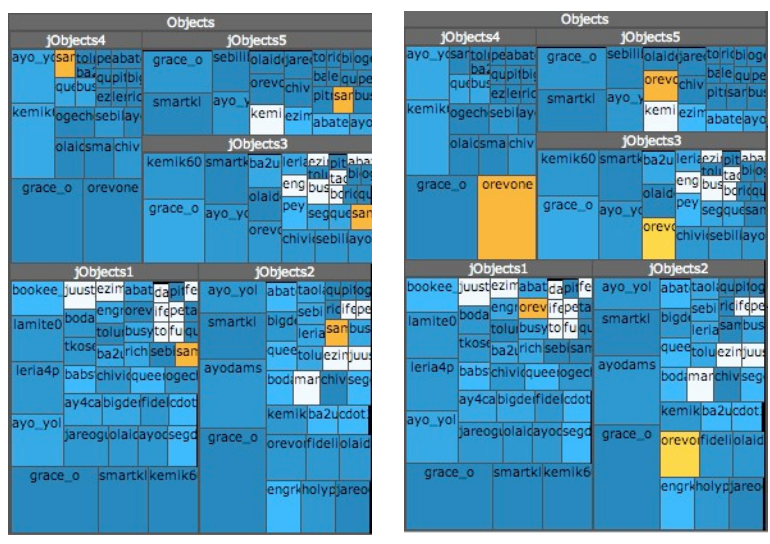

Figure 3. Student A (left); Student B (right).

We conducted a semester-long classroom study with QuizMap. Using log data of students' interactions with OSSM, we compared the usage of self-assessment questions in a non-adaptive course portal without social visualization, QuizGuide, and QuizMap (Table- 1). To our surprise, we found that students who worked with QuizMap made fewer attempts and explored fewer topics than in our earlier systems, which indicated a decrease of engaging power. Yet they achieved a much higher success rate that indicated the impact of the social guidance. We also discovered how the social guidance mechanism does its work: stronger students began to work on problems in QuizMap and left darker and larger tile visual traces among topics for less capable students to follow (Brusilovsky, et al., 2011). However, the decreased engagement was a clear sign of problems and the students' feedback helped us to reveal some of these problems. While students expressed high satisfaction toward visualizing personal and peer performances as well as direct access to content, they felt frustrated with the crowded and cluttered interface. Indeed, as shown on Figure 2 upper right corner, questions from later topics were less likely to be attempted, making the tile sizes very small and thus the whole corner cluttered. Students were also confused by the lack of clear topic progression (TreeMap allocates topics by side, not in order) and the problems with comparing personal and peer knowledge (orange and blue colors were hard to compare). These results motivated us to the next study.

\subsection{Enhancing content organization via Parallel IntrospectiveViews: visualizing peer models through the OSSM interface}

Following QuizMap's experience, we realized that we needed a different representation in order to have a clear picture of content and each individual student model. We selected an appealing IntrospectiveViews interface, which was originally used for visualization of user interest models (Bakalov, et al., 2010) and adapted it to the needs of representing knowledge and social comparison. We called this OSSM interface Parallel IntrospectiveViews (Figure 4) since it offered parallel views of two student models at a time, i.e., student's own models vs. models of their peers or the class as a whole. 

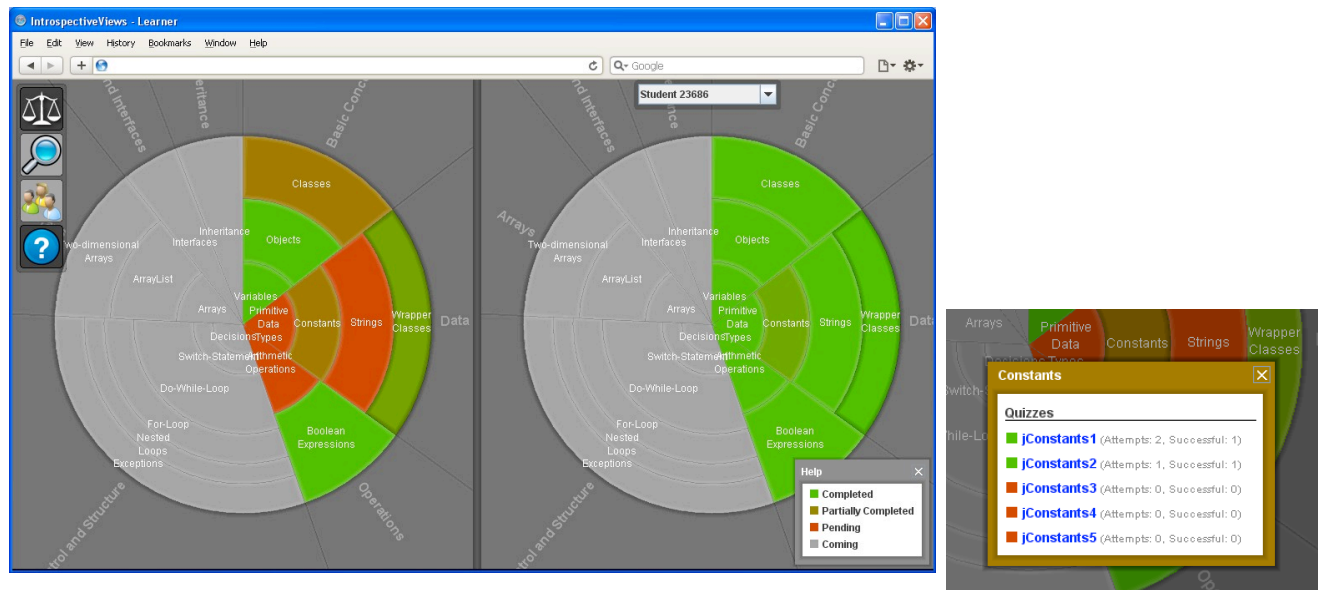

Figure 4. Parallel IntrospectiveViews. Left pane - visualization of the student's own progress; right pane - visualization of a peer's progress. The circular sectors represent the lectures and the annular sectors represent the topics of individual lectures. The shades of the sectors indicate whether the topic has been covered and for the covered ones, denote the progress the student has made. Color screenshots available at: http://www.minerva-portals.de/research/introspective-views/. (left); Parallel IntrospectiveViews. Quizzes of the selected topic (right).

Figure 4 (left) shows the Parallel IntrospectiveViews interface. The visualization consists of two panes: the left pane displays the student's own progress and the right one displays the progress of any class peer or the whole class, whichever is selected from a dropdown menu. Each pane visualizes the respective student's progress as a pie chart. The pie chart representation visually conveys the chronological order of lectures while the size of a sector represents the number of problems for each lecture. A lecture may consist of one or several topics, which are represented as angular segments placed within the circular sector of the corresponding lecture. This representation allows the student to easily estimate the amount of work on each individual topic or lecture, while an apparent topical sequence provides a good picture of progress through the course. In addition to that, the ability to view someone else's progress allows the student to quickly find the peers who can help with a difficult topic or quiz. For example, if the student experiences difficulties in completing some quizzes, using the parallel views, she can find a classmate who has already successfully completed those quizzes to ask for help. Finally, the ability to view the average progress of the entire class allows the student to relate her progress to that of the whole class and estimate whether she is ahead or behind of the class.

We conducted a classroom study to assess the impact of the Parallel IntrospectiveViews OSSM interface on student learning and engagement (again, comparing it against two baselines - the non-adaptive course portal QuizJET and the adaptive JavaGuide). A summary of results is shown in Table- 1. We found a slight increase in student activity in Parallel IntrospectiveViews in comparison with nonadaptive QuizJet, yet not as large as in JavaGuide. It is attributed to the system's strong orientation of comparing personal performance with the class average (which was a default comparison). While the access to social data apparently encouraged less active users to do more work, it could equally discourage stronger students from running too much ahead of the class. As a result, the difference between the most active and least active users is getting smaller. Evidence that this is really happening is the observed $25 \%$ decrease in standard deviations for the number of attempts. In turn, the class as a whole became a bit less adventurous than in the non-social JavaGuide, exploring fewer questions and topics (this is because the variety of topics come to some extent from more 
active users who run ahead of the class). On the other hand, the growth of the success rate demonstrates that knowledge-based and social guidance combined are more effective in guiding the students to appropriate questions that they are ready to handle than knowledge-based guidance alone. The community wisdom does matter.

Parallel IntrospectiveViews followed our earlier systems in providing direct access to learning content through OSSM visualization. Clicking on a topic segment, a student can call a list of problems within this topic that was also socially annotated (Figure 4, right) Moreover, this click could be done on the student's own knowledge map on the left as well on the class/peer knowledge map on the right. Students' logs indicated from which part of the interface content access was made. These logs indicated that students quite actively accessed learning content from their peers' models. This was a strong piece of evidence that students really used the social guidance provided by the interface. Moreover, we found a correlation between the frequency of peer model comparisons and the learning gain. The more the students used social guidance by accessing content on the peer side, the higher post-quiz scores they received $(r=0.34, p=0.004)$. Finally, in the subjective evaluation, students expressed positive gratitude toward the system (Hsiao, et al., 2011). Overall, the results demonstrated a promising impact of social visualization on students' motivation and learning. We were inspired by the outcome and decided to investigate this visualization approach further while enhancing the peer comparison aspect that was apparently efficient.

\subsection{Progressor: Refined OSSM interface for personalized access to programming problems}

The enhanced version of Parallel IntrospectiveViews was named Progressor (Hsiao \& Brusilovsky, 2012). It followed the holistic and easy-to-grasp view of knowledge progress used in the earlier systems while improving access to peer progress data. To achieve the latter goal, we implemented a sortable list of thumbnail previews of student peers' models that replaced earlier "blind" menu. According to the small multiples principle (Tufte, 1990), the thumbnail peer models of the same shapes provide the visual constancy and allow focusing on the differences. To increase the power of social comparison, the visible part of the sorted list displayed the top three performers within the class. We believed that displaying the progress of top students could make the rest of the class more eager to catch up with them than the default comparison with a moderate class average in the earlier system. As before, the user can obtain a detailed view on the progress of any peer by clicking his/her thumbnail and switching to one-to-one comparison mode (Figure. 5-right). In this mode, the user can obtain detailed information about the peer's progress, including the information about the progress on individual quizzes. To balance easy access to peer information, Progressor implemented privacy management. The user can grant and revoke access to his/her progress data for each peer individually. Ultimately, the OSSM design was aligned with the information seeking mantra - "overview first, zoom and filter, details on demand" (Shneiderman, 1996). 

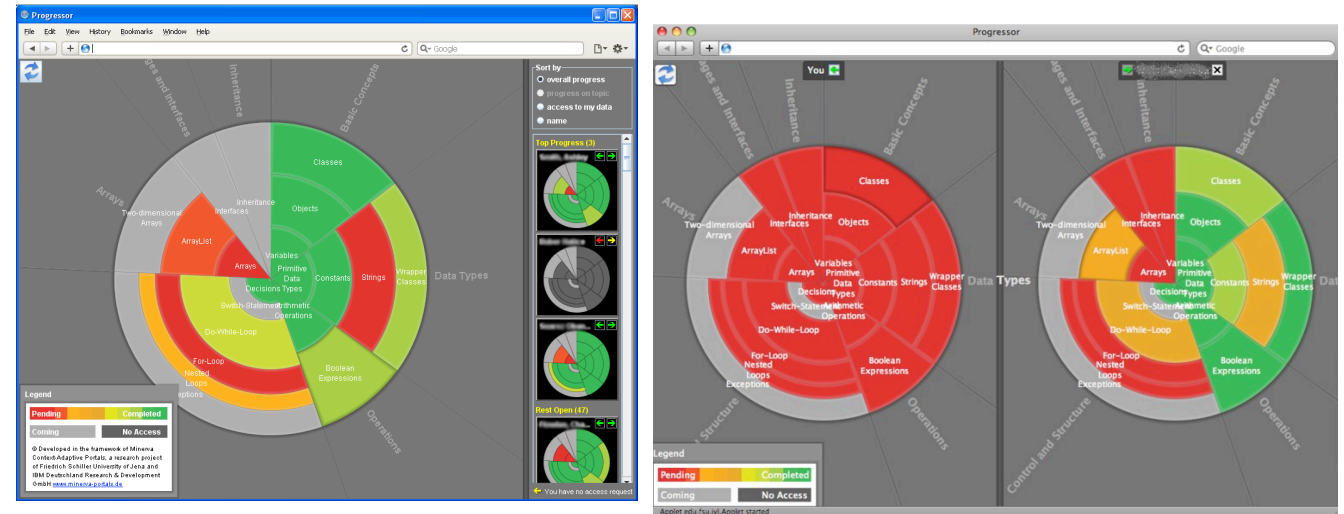

Figure 5. Progressor: Peers' progress are displayed as thumbnails and listed at the side of the user's own model (left); Progressor: Peers model comparison (right).

From a semester-long study cross-compared with previous attempts to organize access to Java problems, we learned that the new design of the OSSM interface was very engaging. Students used Progressor extensively. On average, it achieved the highest system usage across all OSSM interfaces surpassing even the former champion, JavaGuide (Table 1). Progressor also engaged students to explore more topics and to work on more distinct questions. In addition, the amount of time spent on the system (in terms of the sessions) was doubled. However, can we really argue that the boost of usage could be credited to the new design? To answer this question we examined student interaction with the peer side of the Progressor interface such as re-sorting, scrolling, and accessing the peer list. As before, we found that students interacted with the peer side quite considerably, comparing their progress with the progress of peers and accessing a considerable volume of content from the peer side. Moreover, the more students engaged in interacting with the social features of Progressor, the more likely they were to achieve a higher success rate in answering the self-assessment questions. The findings were consistent with the subjective evaluation outcome, which demonstrated high satisfaction with Progressor.

Table 1. OSSM studies statistics summary

\begin{tabular}{|c|c|c|c|c|c|}
\hline & \multicolumn{2}{|c|}{ Baseline } & \multicolumn{3}{c|}{ OSSM } \\
\cline { 2 - 6 } & QuizJET & JavaGuide & QuizMap & PIV & Progressor \\
\hline Attempts & $80.81 \pm 22.06$ & $125.50 \pm 20.04$ & $45.55 \pm 6.67$ & $113.05 \pm 15.17$ & $205.73 \pm 40.46$ \\
\hline $\begin{array}{c}\text { Success } \\
\text { Rate }\end{array}$ & $42.63 \% \pm 1.99 \%$ & $58.31 \% \pm 7.92 \%$ & $79.30 \% \pm 1.94 \%$ & $71.35 \% \pm 3.39 \%$ & $68.39 \% \pm 4.32 \%$ \\
\hline $\begin{array}{c}\text { Distinct } \\
\text { Topics }\end{array}$ & $7.81 \pm 1.64$ & $11.77 \pm 1.19$ & $4.55 \pm 0.59$ & $9.06 \pm 1.39$ & $11.47 \pm 1.34$ \\
\hline $\begin{array}{c}\text { Distinct } \\
\text { Questions }\end{array}$ & $33.37 \pm 6.50$ & $46.18 \pm 5.15$ & $17.07 \pm 2.78$ & $36.5 \pm 5.69$ & $52.7 \pm 6.92$ \\
\hline
\end{tabular}

\subsection{OSSM Design: Lessons Learned}

Through three progressions of OSSM interfaces design and studies, we learned that there are several important features for designing a successful educational social visualization system.

\section{- Interactivity and content access}

Interactivity is very important for OSSM as indicated by the large volume of student interaction with it and the impact of interaction on performance. Interactivity can be implemented in several forms. In our OSSM interfaces, interactivity is implemented by allowing the student to access the learning content directly by clicking on the knowledge 
maps. The idea is simple and effective: the visualization of the user model is not just a widget, but also the main entrance to the learning content. Moreover, students are not only capable of interacting with the content, but they are also enabled to interact with their peers by communicating (requests), comparing and sorting. Other interactivity features are, for example, zooming to allow the user model visualization to deal with complexity and large topic domains (Shneiderman, 1996), and manipulation to allow the user to feel in control over his/her model (Kay, 1997).

\section{- Sequence}

Progressor uses a familiar sequence of lectures and topics (shown clockwise) instead of the random topic allocation used on QuizMap and we believe that it was important for its success. The presented sequence helped students to interpret their progress in the context of their class and provided guidance, yet it doesn't restrict content access: students are allowed to explore ahead or redo already covered topics. Through the series of OSSM studies, we have found that strong students do tend to explore ahead of the class and weak students tend to follow them, even in topics that are beyond the current topic. We think that the ability of the interface to encourage strong students to move ahead of the class is important because it fuels the social navigation mechanism.

\section{- Identity}

Identity representation captures all the information belonging to the student and displays it in a clear form. It makes the students identify themselves with the model and allows them to easily compare with each other $\{$ Bull, $2007 \# 7\}\{$ Chen, $2007 \# 6\}$. We complement these ideas with the concept of unity proposing that the perception of identity is higher if the model represents unity. We believe that Progressor with its clear concentrated representation of students' own knowledge better meets these characteristics than the failed, QuizMap that presented this model in a fragmented manner.

\section{- Easy Comparison}

Letting students compare their knowledge and progress with each other is the key for encouraging more work and better performance (Dijkstra, et al., 2008). Progressor is going further then earlier systems in this direction allowing students to compare themselves with others on two levels, macro- and micro- comparisons. While viewing her/his own model, s/he can see the thumbnails of her/his peers' models. This is the macro-level comparison. It provides high-level comparisons, allowing fast mental overlapping of the colored areas between models. The idea aligns with the small multiples principle (Tufte, 1990), which provides the regularity for drawing attention to the data changes among the peer models. When the student clicks in the peer models, Progressor enters the micro-level comparison mode showing the user and the peer model in full size with greater detail. Both levels of comparisons allow students to perform social comparisons at will.

\section{- Transparency}

Comparison implies model exposure, which directly raises privacy issues. From our survey, there was no single extremely negative opinion regarding privacy and data sharing. Students loved the idea of sharing and comparing. We also found that the students actually had more persistent interactions with the system while they opted for model visibility. While privacy is always an important area in social systems, our study results provided us some insight that the openness of the personal model may be viewed by students positively in our target context.

\section{- Guidance}


Open social student modeling interfaces enable implicit guidance by allowing students to compare with their peers and explicit guidance by showing the best students at the top of the list of thumbnail models. In fact, the results of our studies show that students tended to follow the footprints of the most successful students in the class. This suggests several research opportunities in the future, such as recommender services.

4.

\section{Open Social Student Modeling with Two Kind of Learning}

\section{Content}

The experience with three OSSM systems presented above allowed us to examine the feasibility and the impact of a combined social visualization and open student modeling approach. We also learned several important lessons about the OSSM interface organization to maximize its potential impact. Yet, our earlier systems have one important limitation: they used OSSM to provide personalized access to just one kind of learning content - parameterized programming questions for Java. This can't be considered as a realistic case in the majority of domains. Specifically, in programming language learning, one usually learns from multiple kinds activities and types of learning content, i.e., reading textbooks, exploring program examples, watching videos, writing programs, etc. In addition, more and more kinds of learning resources are made available online.

These considerations defined our next challenge. So far, we have tested OSSM by using one of the representative content collections and summarized a set of important attributes to design effective OSSM. How do we design OSSM interfaces to support many kinds of learning content in parallel? Will current identified design principles (key features) confirm the power of the learning community through OSSM with multiple learning resource collections? Will the OSSM visualization provide successful personalized guidance within a richer collection of educational resources? A more recent study presented in the second part of the paper attempted to answer these questions. In other words, it addressed the OSSM scalability issue and focused on establishing a multi-content OSSM design and explored its impact on students' engagement and learning. In the next two sections we report our attempts to design a multi-content OSSM interface, Progressor ${ }^{+}$, following the principles distilled in our earlier work. We also present the result of a Progressor ${ }^{+}$evaluation.

\subsection{System Design: Progressor ${ }^{+}$: an advanced OSSM interface for multiple content collections}

The goal of Progressor ${ }^{+}$was to bring our earlier findings up to scale and explore the feasibility of open social student modeling in the context of more diverse learning content. To achieve this goal, we designed a new scalable tabular interface to accommodate diverse content. Figure 6 shows a conceptual diagram on the progression of OSSM interfaces and content coverage. 


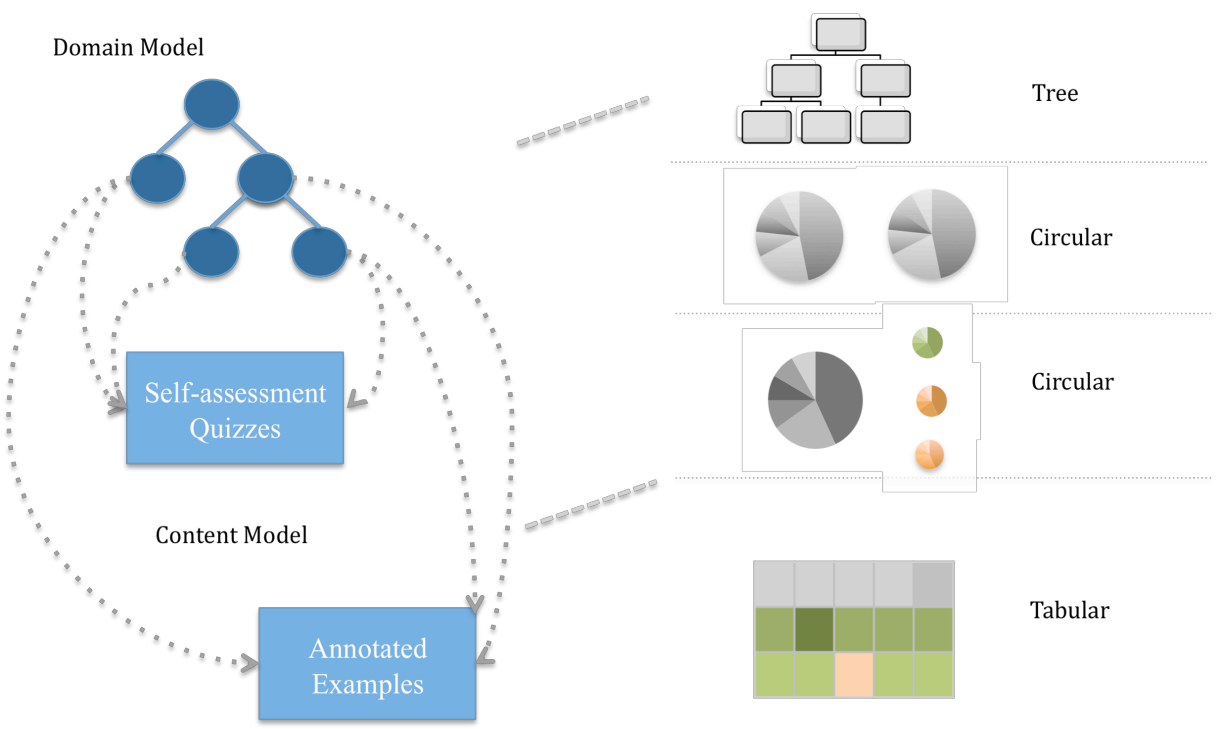

Figure 6. Open social student modeling interfaces progression and content coverage.

We transferred all the features from the three earlier systems into the new design of Progressor ${ }^{+}$. The interface is presented in Figure. 7. Each student's model is represented as several rows of a large table with each row corresponding to one kind of learning content and each column corresponding to a course topic. We incorporate two sizable pools of learning content in Progressor ${ }^{+}$- parameterized self-assessment questions and annotated code examples (thus Figure 7 shows two rows for each student - a quiz progress row and an example progress row), however, the tabular nature of the Progressor ${ }^{+}$interface allows adding more kinds of content when necessary. Each cell is colored coded to show the student's progress on the topic. We used a ten-color scheme to represent percentiles of the progress (Figure 7). The use of color-coding allows collapsing table rows that are out of focus, thus making it possible to present a progress picture of a large class in a relatively small space. This feature was inspired by the TableLens visualization, which is known as highly expressive and scalable (Rao \& Card, 1994).

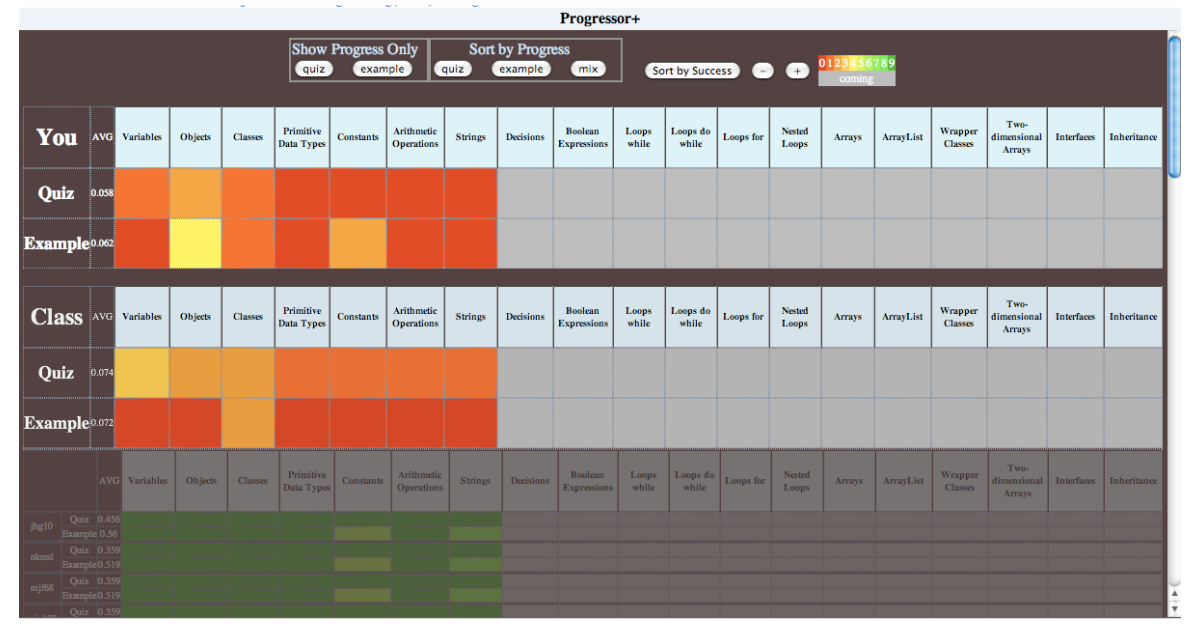




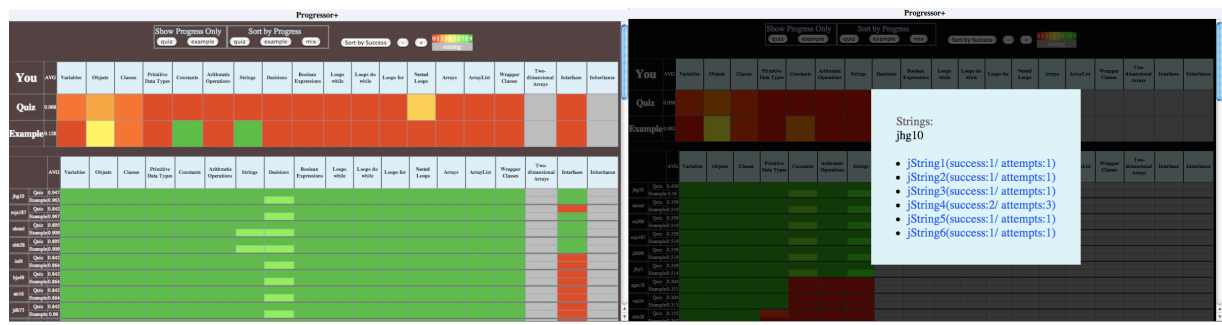

Figure 7. Progressor ${ }^{+}$: the tabular open social student modeling visualization interfaces.

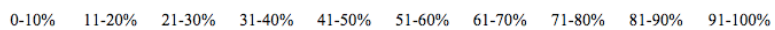

Figure 8. Ten-color shades representation of the summarized progress analytics.

Essentially, all the rows are joined together and are presented in a single large table. In other words, all the student models are combined in the same big table. There are several other table layout options available for students, including a collapsed view, an expansion view and a filtered view. The collapsed and expansion views are used to focus on the target student model or the specific type of content. Students are able to manipulate the views for model comparisons or detail inspections. The filtered view requires a criterion selection to refine the exploration view. The filtering criteria include sorting the progress by content types and sorting by success rate. The default setting of Progressor+ is configured as fully expanded table rows of the whole community and sorted by average progress in descending order. To access the content, students interact directly with the Progressor+ table cells by clicking on the intersection of the topic and the content type (Figure 7 - right). Once this is selected, a panel of the lists of content will be presented along with usage details for each content item. For instance, how many attempts have there been on the question? How many times has the question been successfully solved? How many lines of annotations have been studied?

\subsection{Study design and procedure}

To achieve the objectives of this work, we designed a semester-long classroom study by providing the system as one of the supplemental course tools for the class. A semester-long classroom implementation will allow us to obtain a realistic longer-term case of the technology compared to the regular 2 hour lab study. It will also capture a more realistic scenario of the curriculum on all ranges of course topics. More importantly, it will allow us to measure the long-term student engagement. To validate the hypotheses, the study will be compared to three other classroom studies. All three other classroom studies featured the same classes, same kinds of students, same course materials (including textbooks, slides, assignments, exams), same course schedule, same pre-/post- tests and same set of self-assessment questions and annotated examples.

The classroom studies were carried out in the undergraduate course "Fundamentals of Object-Oriented Programming" offered at School of Information Sciences at the University of Pittsburgh. This is a required course for the Information Science major. The students registered for this course were commonly a mixture of students with Information Sciences majors and undeclared students from the School of Arts and Sciences. Only a few students from other sciences or engineering related degree programs registered for this course. QuizJET was introduced in the 2008 Spring semester; JavaGuide was introduced in the 2008 Fall semester; Progressor was introduced in the 2011 Spring semester and Progressor ${ }^{+}$was introduced in the 2012 Spring semester.

The Progressor conditioned semester is consider as the primary baseline group, 
because the instructor was the same as the one in the Progressor ${ }^{+}$case. Due to QuizJET and JavaGuide semesters were taught by different teachers, therefore, they are considered as secondary baselines. It is essential to point out that the systems were used as non-mandatory tools for the course. In this work, we consider the groups of students who used the systems as the sample of volunteer subjects. Table 1 shows the composition of the conditions and the participants of all the classroom studies, including the number of students, male and female composition, weak and strong distribution, and average scores in the pre-tests.

Table 2. Study conditions \& participants

\begin{tabular}{|c|c|c|c|c|}
\hline & \multicolumn{4}{|c|}{ Conditions } \\
\hline & \multicolumn{2}{|c|}{ Secondary Baselines } & Primary Baseline & Experiment \\
\hline Semester & 2008 Spring & 2008 Fall & 2011 Spring & 2012 Spring \\
\hline Systems & QuizJET & JavaGuide & Progressor & Progressor $^{+}$ \\
\hline Content & Quizzes $^{1}$ & Quizzes $^{1}$ & Quizzes & $\begin{array}{c}\text { Quizzes, } \\
\text { Examples }\end{array}$ \\
\hline \multicolumn{5}{|c|}{ Number of the students } \\
\hline Overall & 31 & 38 & 51 & 56 \\
\hline $\begin{array}{l}\text { Working with } \\
\text { the system }\end{array}$ & $16(52 \%)$ & $22(58 \%)$ & $30(59 \%)$ & $38(68 \%)$ \\
\hline \multicolumn{5}{|c|}{ Male/Female student distribution } \\
\hline Overall & $25 / 6$ & $27 / 11$ & $36 / 15$ & $44 / 12$ \\
\hline $\begin{array}{l}\text { Working with } \\
\text { the system }\end{array}$ & $13 / 3$ & $16 / 6$ & $23 / 7$ & $32 / 9$ \\
\hline \multicolumn{5}{|c|}{ Weak / Strong student distribution } \\
\hline Overall & $16 / 15$ & $30 / 8$ & $41 / 10$ & $49 / 7$ \\
\hline $\begin{array}{l}\text { Working with } \\
\text { the system }\end{array}$ & $6 / 9$ & $14 / 5^{2}$ & $26 / 4$ & $34 / 4$ \\
\hline \multicolumn{5}{|c|}{ Average scores in pre-test } \\
\hline Overall & 10.18 & 4.97 & 3.53 & 3.20 \\
\hline $\begin{array}{l}\text { Working with } \\
\text { the system }\end{array}$ & 10.20 & 2.68 & 3.67 & 3.05 \\
\hline \multicolumn{5}{|c|}{ IS majored / others (undeclared, mechanical engineering, biomedical informatics) } \\
\hline Overall & $25 / 6$ & $21 / 17$ & $23 / 28$ & $23 / 33$ \\
\hline $\begin{array}{l}\text { Working with } \\
\text { the system }\end{array}$ & $12 / 4$ & $10 / 12$ & $8 / 22$ & $17 / 21$ \\
\hline
\end{tabular}

All four classes were given the same pre-test during the first week to collect their preknowledge of the course. The systems were introduced to the classes at the third week of each semester and were available for the students from then on, for an overall fifteen week time period. During the fifteen weeks, students voluntarily logged onto the systems and worked on the QuizJET exercises or/and the WebEx examples. Students were instructed on how to use the systems and advised to use the systems but such use was not mandatory for the course work. The post-tests were administrated at the $16^{\text {th }}$ week of the classes to measure the students' learning. A questionnaire survey was given shortly after the post-tests. There were four exams including the final exam across each semester; they were the important evaluation time marks and scheduled at the 5th, 9th, 15th and 17th week of the semester accordingly. To ensure that the student cohorts were comparable, we first examined the students' pre-test scores. A one-way between-subjects analysis of variance was performed on the pre-test scores as a function of 4 different

\footnotetext{
${ }^{1}$ Examples were also available to the class through a traditional course management portal instead of having the navigational support through the social visualization interface

${ }^{2}$ Three students working with the system in the Fall 2008 semester did not take the pre-test.

3 That the students who used QuizJET had significant higher pre-tests scores could be attributed to two reasons. 1) there were stronger students used the system that term. 2) there were more Information Sciences majored students using the system. 3) there were more repeaters from previous semester, which had already been given the pre-tests once.
} 
interfaces (QuizJET, JavaGuide, Progressor, and Progressor ${ }^{+}$). We found the students who used the QuizJET $(M=10.20, S E=0.048)$ system had significantly higher preknowledge than the average of the other three systems $(M=3.13, S E=0.048), F(3,99)=$ $3.258, p=0.0253$. The assumption of homogeneity of variance was met, Brown-Forsythe $F(3,99)=2.750, p=.052$. The assumption of normality was only met for the QuizJET group (Table 2 ).

Table 2. Test of normality of the pre-test scores for each system

\begin{tabular}{|c|c|c|c|}
\hline System & Shapiro-Wilk $W$ & $d f$ & $p$ \\
\hline QuizJET & .923 & 16 & .186 \\
\hline JavaGuide & .816 & 22 & .002 \\
\hline Progressor $^{+}$ & .838 & 30 & .000 \\
\hline Progressor $^{+}$ & .897 & 38 & .002 \\
\hline
\end{tabular}

Table 3. Summary of all parameter statistics of self-assessment quizzes collection

\begin{tabular}{|c|c|c|c|c|c|}
\hline \multicolumn{6}{|c|}{ Quiz } \\
\hline & Parameters & QuizJET & JavaGuide & Progressor & Progressor $^{+}$ \\
\hline & $\begin{array}{l}\text { Active } \\
\text { users }\end{array}$ & 16 & 22 & 30 & 38 \\
\hline \multirow{3}{*}{ Average } & Attempt & $80.81 \pm 22.06$ & $125.5 \pm 25.66$ & $205.73 \pm 40.46$ & $190.42 \pm 21.20$ \\
\hline & Success & $\begin{array}{c}42.63 \% \pm 1.99 \\
\%\end{array}$ & $\begin{array}{c}58.31 \% \pm 2.74 \\
\%\end{array}$ & $\begin{array}{c}68.39 \% \pm 4.32 \\
\%\end{array}$ & $\begin{array}{c}71.20 \% \pm 4.49 \\
\%\end{array}$ \\
\hline & Session & $3.75 \pm 0.53$ & $4.14 \pm 0.65$ & $8.4 \pm 1.39$ & $5.18 \pm 0.55$ \\
\hline \multirow[b]{2}{*}{ Coverage } & $\begin{array}{l}\text { Distinct } \\
\text { topics }\end{array}$ & $7.81 \pm 1.64$ & $11.77 \pm 1.07$ & $11.47 \pm 1.34$ & $12.92 \pm 0.90$ \\
\hline & $\begin{array}{l}\text { Distinct } \\
\text { question }\end{array}$ & $33.37 \pm 6.50$ & $46.18 \pm 6.11$ & $52.70 \pm 6.92$ & $61.84 \pm 4.49$ \\
\hline
\end{tabular}

Table 4. Summary of all parameter statistics of annotated examples collection

\begin{tabular}{|l|l|c|c|c|c|}
\hline & Parameters & QuizJET & JavaGuide & Progressor & Progressor $^{+}$ \\
\hline & Active users & 21 & 20 & 7 & 35 \\
\hline \multirow{5}{*}{ Average } & Example & 10.86 & 19.75 & 28.71 & 27.37 \\
\cline { 2 - 6 } & Line & 104.24 & 116.6 & 219.71 & 184.18 \\
\cline { 2 - 6 } Coverage & Session & 4.42 & 5.35 & 5.50 & 4.94 \\
\cline { 2 - 6 } & $\begin{array}{l}\text { Distinct } \\
\text { topics }\end{array}$ & 8.48 & 9.15 & 12.28 & 12.20 \\
& $\begin{array}{l}\text { Distinct } \\
\text { examples }\end{array}$ & 10.86 & 17.3 & 25.125 & 27.37 \\
\cline { 2 - 6 } & $\begin{array}{l}\text { Distinct } \\
\text { lines }\end{array}$ & 80.33 & 67.1 & 115.22 & 141.5 \\
\hline
\end{tabular}

We summarize the differences between the conditions and the main direction of the effects of this work that we anticipated discovering for both collections of content (Figure 9). In Table 3 and Table 4, we present all the parameters' average statistics for both content collections in all the conditions. The table will be broken down and dissected in detail in the following subsections: 1) The impact on motivation and engagement; 2) The impact on students' learning 3) The social mechanism; 4) The subjective evaluation. 


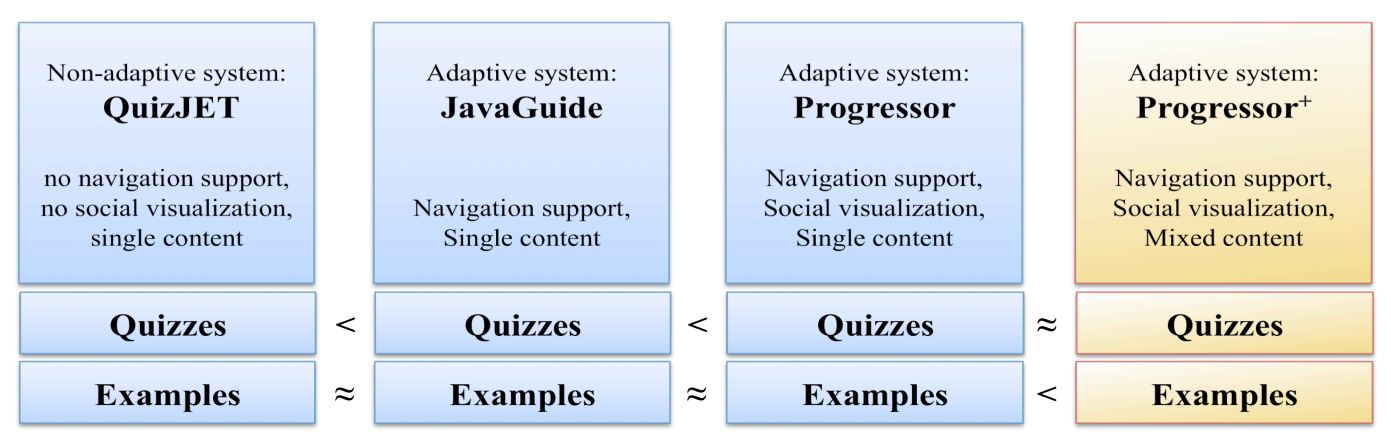

Figure 9. Expected effects of the conditions

\subsection{Outcome variables}

We follow our prior work in examining OSSM interfaces and students' learning effects; a set of variables and measurements are in Table 5.

Table 5. Definitions for parameters used

\begin{tabular}{|c|c|}
\hline Parameter & Definition \\
\hline Questions & $\begin{array}{l}\text { Number of questions that a student attempts to } \\
\text { solve }\end{array}$ \\
\hline Success rate & $\begin{array}{l}\text { Number of questions correctly answered divided } \\
\text { by all attempts }\end{array}$ \\
\hline Examples & Number of examples that a student explores \\
\hline Lines & Number of lines that a student explores \\
\hline Exploration rate & $\begin{array}{l}\text { Number of lines explored divided by all explored } \\
\text { example lines }\end{array}$ \\
\hline Topic coverage & Distinct number of topics viewed \\
\hline Question coverage & Distinct number of questions attempted \\
\hline Example coverage & Distinct number of examples explored \\
\hline Line coverage & Distinct number of lines explored \\
\hline
\end{tabular}

Using these variables and other data we have developed several ways to measure the expected outcome. The outcome measurements are discussed below:

- Motivation \& Engagement:

In investigating students' motivation and engagement, we hypothesize that students are motivated and engaged in using Progressor ${ }^{+}$and produce more quantities of interactions and higher coverage. Specifically, we expect the Attempts, Time and the diversity of the content explored will increase.

First of all, we summarize the systems' usage to gauge the students' motivation and engagement. The independent variables include the question Attempts, the explored examples, the explored example lines, the course coverage (distinct topics, distinct questions and distinct examples) and the time spent on interacting with the systems.

Secondly, following the topic-based personalization guidance, students are expected to focus on the "current" topics (Zone A - lecture stream zone in Figure 11) (Brusilovsky, et al. 2009). In Figure 18, the shaded areas in Zone C \& D are the regions of the off-"current" course topic activities, which are the self- motivated activities performed by the students themselves. Thus, we measure the ratio of 
students' activity performed outside the current course focus to the topic coverage that a student roams and works with in the system. The computational notation is presented in Equation 1, where $m$ is denoted as motivation and $i$ stands for each student. We called this indicator the M-ratio. To better understand the depth of the intensity of students' motivation, such a ratio can be further divided into two statistics, forward $r_{m}$ and backward $r_{m}$, where forward $r_{m}$ represents the ratio of moving ahead of the current course focus and backward $r_{m}$ represents revisiting past topics. Both statistics explain the students' self-motivation to work on the content through the systems. The canonical formula is presented in Equation 2. For the M-ratio, we used the number of actions in the Zone $\mathrm{C} \& \mathrm{D}$ divided by the total number of actions. To calculate the measure of forward $r_{m}$, we used Zone A \& D, where we used Zone A \& $\mathrm{C}$ to calculate the measure of backward $r_{m}$.

Equation 1: M-ratio

$$
r_{m}=\frac{\text { \#outsideScope }_{\text {Topic }}}{\text { \#attemptedTopic }_{i}}
$$

Equation 2: the canonical M-ratio

$$
r_{m}=\text { forward }_{r_{m}}+\text { backward }_{r_{m}}
$$

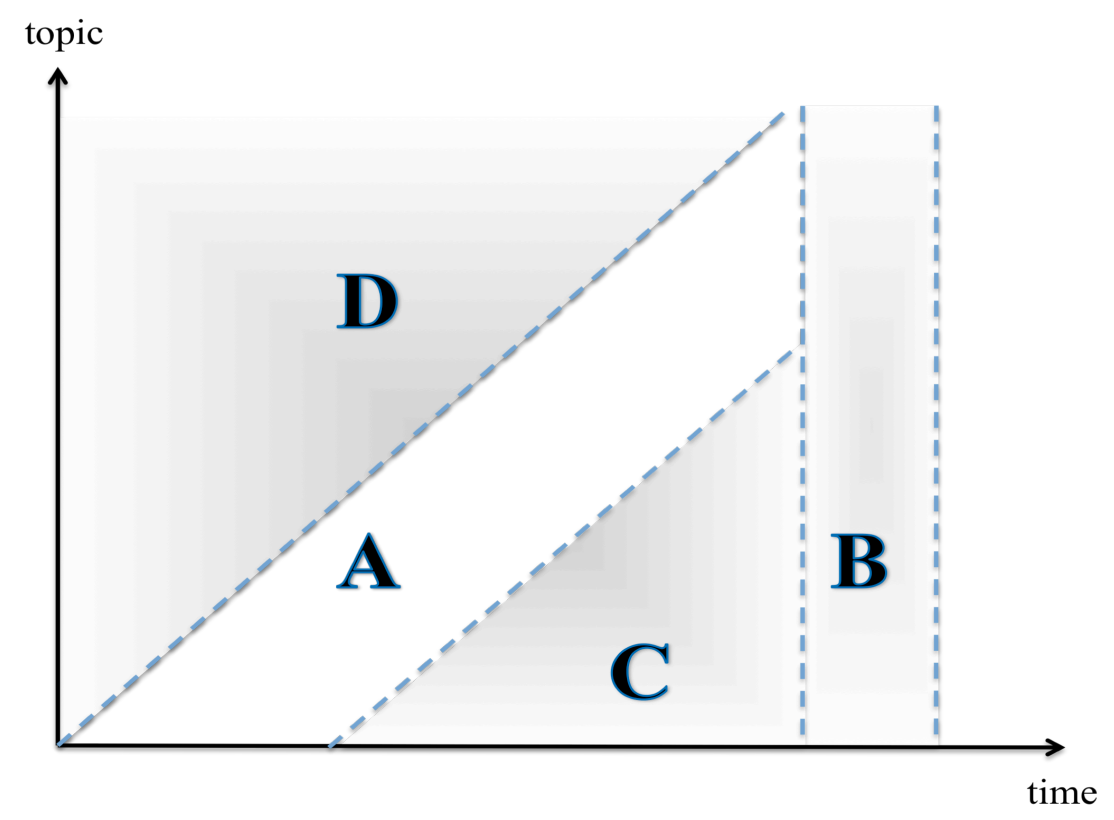

Figure 11. Projected self-motivated activities

\section{- Learning:}

In investigating students' learning results, we hypothesize that students will benefit from Progressor $^{+}$and result in higher absolute knowledge gain. Meanwhile, we expect multiple collections of content will result in the highest normalized knowledge gain. Therefore, we use pre-test and post-test scores to measure the students' knowledge gain. The canonical formula of the student's Absolute Knowledge Gain is denoted as the differences between pre-test and posttest scores (Equation 3). The normalized knowledge gain, also be computed based on Equation 4.

Equation 3: Absolute knowledge gain 
Equation 4: Normalized knowledge gain

Normalized Knowledge Gain $=\quad \frac{\text { PosttestScore }- \text { PretestScore }}{1-\text { PretestScore }}$




\section{Evaluation Results}

\subsection{Impact on motivation and engagement}

If we can demonstrate that there is no significant difference in the amount of work done between Progressor and Progressor ${ }^{+}$and that both are significantly higher than the non-adaptive system, QuizJet, we will conclude that Progressor ${ }^{+}$will work in a scalable content framework. We performed a one-way between-subjects analysis of variance on the quantity of the work done as a function of system conditions. Table 5 summarizes the test results of two collections of work for three conditions. As we anticipated, we did not find significant differences in the amount of work done between Progressor and Progressor ${ }^{+}$. Progressor ${ }^{+}$worked as well as Progressor. To prove that adaptive navigation support combined with the social visualization approach will work for a mixed collection of educational content, we have to show that this approach supports more educational activities than the non-adaptive system. The statistical analysis showed that, indeed, for both Progressor and Progressor ${ }^{+}$students completed significantly higher amounts of work (questions, examples and lines) than for QuizJET, which verified that our approach motivated the students to put more effort in working with the systems.

Table 5. The statistics for comparing the amount of work done among systems

\begin{tabular}{|c|c|c|c|}
\hline & & F-stats & $p$-value \\
\hline \multirow{2}{*}{ questions } & $\begin{array}{l}\text { QuizJET }(M=80.81, S E=27.13) \\
\text { vs. } \\
\text { Progressor }(M=205.73, S E=27.13)\end{array}$ & $F(1,44)=24.20$ & $<0.001$ \\
\hline & $\begin{array}{l}\text { QuizJET }(M=80.81, S E=27.13) \\
\text { vs. } \\
\text { Progressor }^{+}(M=190.42, S E=27.13)\end{array}$ & $F(1,52)=23.72$ & $<0.001$ \\
\hline \multirow{2}{*}{ examples } & $\begin{array}{l}\text { QuizJET }(M=10.86, S E=4.22) \\
\text { vs. } \\
\text { Progressor }(M=28.71, S E=4.22)\end{array}$ & $F(1,26)=12.13$ & $<0.001$ \\
\hline & $\begin{array}{l}\text { QuizJET }(M=10.86, S E=4.22) \\
\text { vs. } \\
\text { Progressor }^{+}(M=27.37, S E=4.22)\end{array}$ & $F(1,54)=11.89$ & $<0.001$ \\
\hline \multirow{2}{*}{ lines } & $\begin{array}{l}\text { QuizJET }(M=104.24, S E=21.32) \\
\text { vs. } \\
\text { Progressor }(M=219.71, S E=21.32)\end{array}$ & $F(1,26)=9.55$ & $<0.001$ \\
\hline & $\begin{array}{l}\text { QuizJET }(M=104.24, S E=21.32) \\
\text { vs. } \\
\text { Progressor }^{+}(M=184.18, S E=21.32)\end{array}$ & $F(1,54)=7.11$ & 0.007 \\
\hline
\end{tabular}

Motivating students to do more work should lead to better performance. To confirm that the motivational effects of the adaptive navigational support and social visualization actually led to more positive outcomes, we examined the Course Coverage parameters and performed correlation analyses. To begin, we found that students worked significantly more distinct questions, studied more examples and lines in the combined approach systems than with no support at all. The Pearson correlation coefficient indicated that the more diverse the questions the students tried, the higher success rate they obtained $(r=0.707, p<.01)$ and the more diverse the examples the students studied, the higher success rate they obtained $(r=0.538, p<.01)$. We also looked at how frequently the students repeated the questions, examples and lines. We found that the more the students repeated the same questions and the more the students repeated studying the same lines the higher success rate they obtained $(r=0.654, p<.01$; $\mathrm{r}=0.528, \mathrm{p}<.01)$. The analysis of motivational effects presented in this section has suggested that the combined approach can effectively enhance students' motivation in the targeted learning context. In the next section, we continue analyzing the effects of such an approach on students' engagement.

In our pre-studies (section 3.3), we found that students doubled the time spent (in terms of sessions) in Progressor compared to QuizJET. However, we did not find this pattern in the 
same parameter when comparing Progressor ${ }^{+}$and QuizJET. Nevertheless, the intensity of students' work per session is actually higher in Progressor ${ }^{+}$. This suggested students might be spending more time in Progressor ${ }^{+}$than in QuizJET but in fewer sessions. Therefore, we computed the actual average time spent for each content collection (Table 7). The results showed that students spent fewer sessions in Progressor ${ }^{+}$in quizzes. However, they did work longer per session. On average, they spent 3.72 and 4.94 times more minutes in Progressor and Progressor $^{+}$than in QuizJET. There were no significant differences between Progressor and Progressor $^{+}$in total time spent on the quizzes. From the example collection, we found that students spent 4.13 and 3.23 times more minutes in studying the annotated examples in Progressor $^{+}$than in QuizJET and Progressor. These differences were both significant. With the adaptive navigation support and social visualizations combined, students studied more. Overall, each student averaged nearly 5 hours of work on the quizzes in Progressor ${ }^{+}$and 5 hours 20 minutes to study the annotated examples. These numbers alone demonstrated that our approach successfully engaged students in working on these non-mandatory systems. In addition, we found that the more time the students spent in one type of content in Progressor ${ }^{+}$, the more likely they were to spend more time in another type of content $(r=0.81, p<.01)$. Yet, does longer engagement lead to better learning? We will discuss the effects on students' learning in the next section.

Table 7. The intensity measures of students' work for all conditions

\begin{tabular}{|c|l|r|r|r|r|}
\hline Intensity & & QuizJET & JavaGuide & Progressor & Progressor+ \\
\hline \multirow{3}{*}{ Quiz } & $\begin{array}{l}\text { Time/session } \\
\text { (minutes) }\end{array}$ & 16.01 & 36.28 & 26.75 & $57.32^{* *}$ \\
\cline { 2 - 6 } & $\begin{array}{l}\text { Total time } \\
\text { (minutes) }\end{array}$ & 60.04 & $150.19^{* *}$ & $224.7^{* *}$ & $296.9^{* *}$ \\
\cline { 2 - 6 } Example & $\begin{array}{l}\text { Attempt/session } \\
\text { Time/session } \\
\text { (minutes) }\end{array}$ & 21.55 & 30.31 & 24.49 & 36.73 \\
\cline { 2 - 6 } & $\begin{array}{l}\text { Total time } \\
\text { (minutes) }\end{array}$ & 69.52 & 121.23 & 110.66 & $321.1^{* *}$ \\
\cline { 2 - 6 } & Example/session & 2.45 & 3.69 & 4.56 & 5.54 \\
\cline { 2 - 6 } & Lines/session & 23.54 & 21.79 & 34.95 & 38.69 \\
\hline
\end{tabular}

\subsection{Impact on student' learning}

Our approach to educational innovation is not complete without an analysis of its impact on students' learning. Our approach demonstrated an impressive motivational and engagement effect on students. However, we are mindful that the students might learn a subject in many many ways, (e.g., labs, lectures, assignments). To demonstrate the efficacy of our approach, we need to show that students' activities with the systems were transformed into learning gains. Therefore, in this subsection, we focus on the association of students' interactions with Progressor $^{+}$and their learning results. We consider the results of pre- and post- tests scores to determine general learning gains.

We performed paired sample t-tests to evaluate the significance of the students' Absolute Knowledge Gain. We found that students who used Progressor ${ }^{+}$indeed achieved post-test scores $(M=15.0, S D=0.6)$ that were significantly higher than their pre-test scores $(M=3.2$, $S D=0.5), t(37)=17.276, p<.01$. In addition, we performed a one-way between-subjects analysis of variance on the Normalized Knowledge Gain as a function of 4 different systems (QuizJET, JavaGuide, Progressor and Progressor ${ }^{+}$). We found that students obtained a significant Normalized Knowledge Gain by working on the self-assessment questions through Progressor ${ }^{+}$ $(\mathrm{M}=0.581, \mathrm{SE}=0.050)$ compared to QuizJET $(M=0.361, S E=0.050), F(1,52)=4.223, p<.05$, $\eta^{2}=.025$. Following previous motivational and engagement analyses, we also found that the more the students studied (more lines), the more knowledge they gained $(r=0.492, p<.01)$. Themore time the students spent on the content (quizzes and examples), the more knowledge they 
gained $(r=0.563, p<.01 ; r=0.448, p<.01)$.

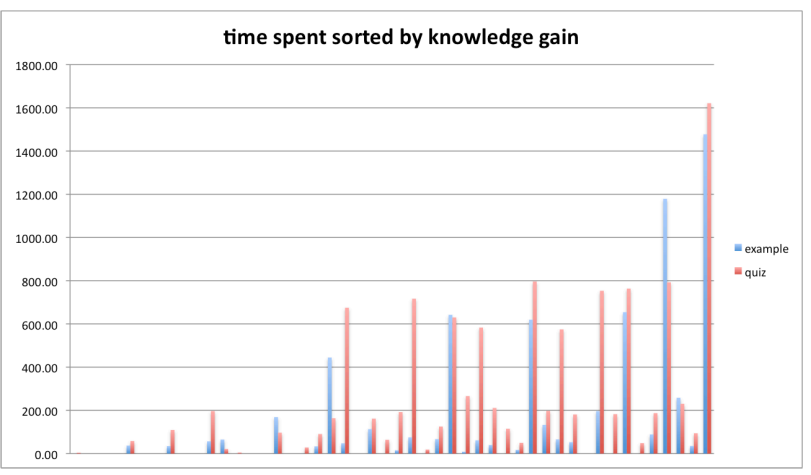

Figure 10. Students' time spent on both examples and quizzes in Progressor ${ }^{+}$sorted by the knowledge gain

\subsection{The mechanism of social guidance}

Our study demonstrated that social guidance could match or even surpass traditional knowledge-based guidance in its ability to guide students to the right content in the right time. But what is the mechanism of social guidance? Why is the progress data collected from the class and presented in visual form able to provide this remarkable quality of guidance, matching guidance based on expert knowledge? That was an important mechanism of our approach to provide social guidance where stronger students would leave traces for weaker ones to follow. However, that pattern was only found within the context of self-assessment quizzes. Do we find the same pattern among multiple collections of educational resources? Are the stronger students still capable of pioneering a good route for the class? Are there any other social mechanisms and effects derived from Progressor ${ }^{+}$? In this subsection, we summarize the findings of social visualizations and plot the system interactions for pattern discovery.

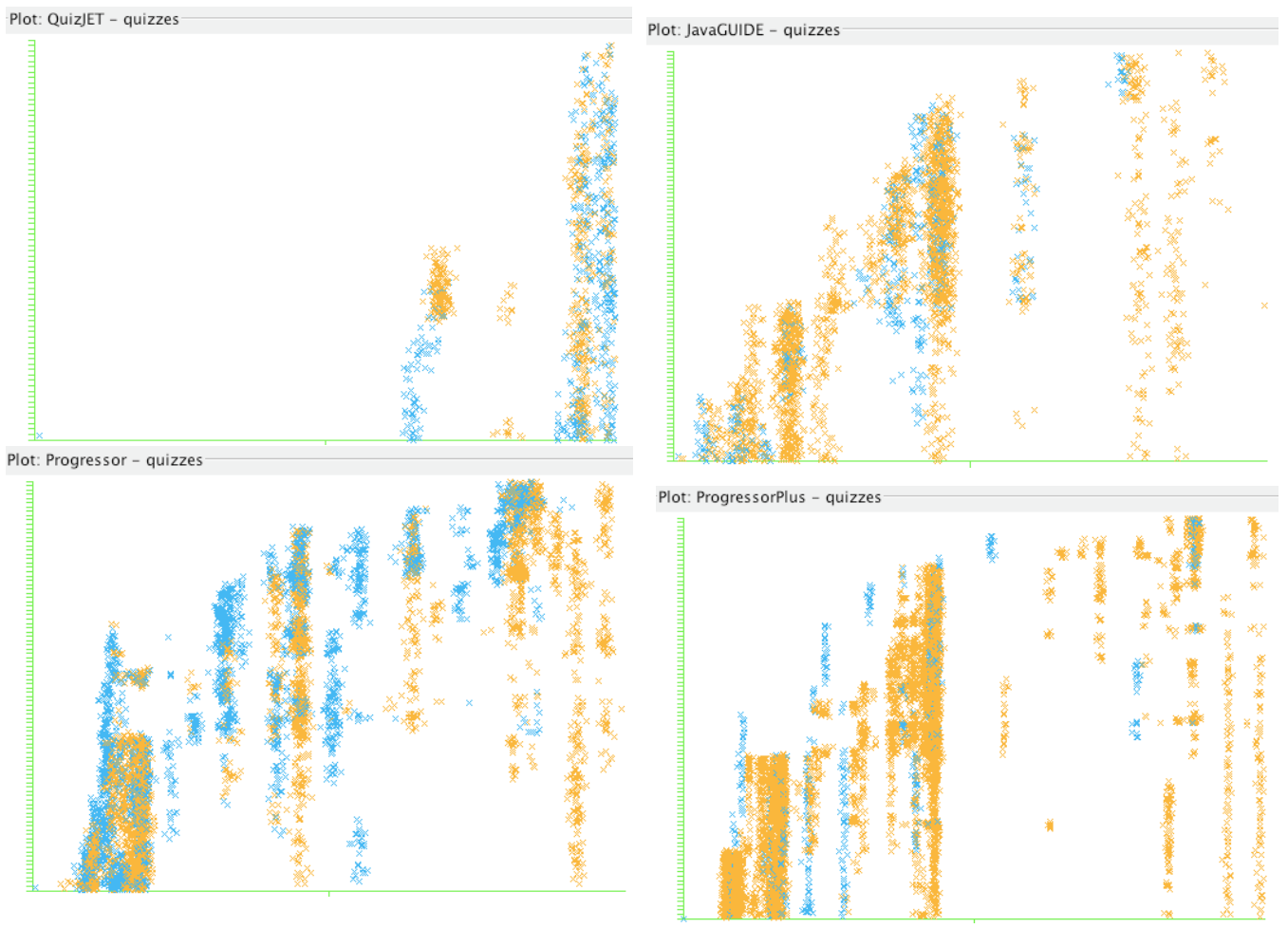

Figure 11. All quizzes attempts distribution by time and question complexity performed by the students in four systems. top-left: QuizJET(a); top-right: JavaGuide(b); bottomleft: Progressor(c); bottom-right; Progressor+(d)

In Figure 11, we plotted all the students' activities on the four systems (QuizJET, JavaGuide, Progressor and Progressor ${ }^{+}$) by time and question complexity. The time of the 
interaction is marked on the $\mathrm{X}$-axis and the question complexity goes from easy to complex on the Y-axis. Each data point represents an attempt at a question. The blue dots belong to the stronger students and the oranges ones belong to the weaker ones. By visualizing all the interactions performed on the systems, we observed several interesting findings.

- There is a general pattern for all conditions, which is that the students were found actively working with the systems during exam preparation periods. They tended to work on the topics from past to current. During the final exam period, students tended to review the full range of the topics. Due to the fact that the subject is inherently cumulative in nature, we expect to find this pattern as a stable effect.

- With topic-based personalization (b\&c\&d), there were noticeable trends indicating that students progressed, which resulted in more work done according to the lecture stream. This is an important message that students were focusing and beyond the current scope. Without such personalization (a), students were only found to work on the systems for exam preparation, yielding a very skewed distribution of Attempts.

- Differences in the amount of work (Attempts) were noticeable from the two figures ( $a \& b)$ on the top row to the bottom two figures (c \& d). The bottom two figures (c $\&$ d) represent the systems with the influence of social visualization, which resulted in a higher intensity of attempts. This not only demonstrated that the students were voluntarily engaging with the systems, but also showed the consistency of the motivational effect over time.

- $\quad$ The timing for beginning work in the system was also revealed by the differences of pre-knowledge levels with the social visualization mediation, where the pre-knowledge levels were determined by the pre-test scores (ranging from a minimum of 0 to a maximum of 20, with the threshold at score 7); strong students scored 7 points or higher (7 13) and weak students scored less than $7(0 \sim 6)$. The strong students tended to explore the questions ahead of the weaker ones (the blue dots go before the orange dots) in social visualization systems ( $c \& d$ ). In Table 10, we calculated the average time that the strong students attempted the question before the weak students did across all ranges of question complexities. On average, strong students worked on the questions 38.04 and 37.70 hours in advance of the weaker students. The effect was much more noticeable for the Complex questions. This indicates a useful pattern of implicit social guidance that stronger students left traces for weaker students to follow. Without the social guidance, there were no clear patterns found (a \& b). Strong and weak students' actions were mixed. Strong ones may be under challenged, while the weak ones may suffer from venturing too fast toward advanced questions.

- A model exposure difference was found between the two social visualization systems (c \& d). Both Progressor and Progressor ${ }^{+}$users were exposed to the entire model, from each individual's to the class. However, the pie shaped model in Progressor took a relatively bigger portion of the space on the screen compared to the table model in Progressor+. The model thumbnails preview was limited by the screen sizes and resulted in presenting only the top students from the class at a first glance in Progressor. Students had to scroll down the sorted model list to see the rest of the models. In Progressor+, on the other hand, there was less scrolling required to view the complete model list. In other words, the top students' models seemed to stand out as highlighted models in Progressor. This may have given extra incentive for the top students, which resulted in encouraging competitiveness and hard work. Therefore, the model exposure differences explained why the stronger students in Progressor tended to work more than in Progressor+.

Table 10. Average Number of Hours that Stronger Students Attempted to Answer Questions Before Weaker Students Attempted to Answer Questions by Question Complexity and for the Progressor and Progressor+ Systems

\begin{tabular}{|c|l|c|c|c|}
\hline (hours) & Easy & Moderate & Complex & Average \\
\hline Progressor & 17.15 & 13.39 & 83.59 & 38.04 \\
\hline Progressor $^{+}$ & 9.17 & 19.63 & 84.30 & 37.70 \\
\hline
\end{tabular}




\subsection{Subjective Evaluation}

In addition to the log analysis, we distributed questionnaires to collect students' opinions of the Progressor ${ }^{+}$system at the end of the classroom study. There were 24 students who filled out the survey, 17 male and 7 female. In the survey, there were 23 questions, ranging from the usability of GUI elements to the users' satisfaction with the interface in general. Users were asked to evaluate the questions on a 5-points Likert scale, 1 - Strongly Disagree; 2 Disagree; 3 - No Strong Opinion; 4 - Agree; 5 - Strongly Agree. They were also advised to provide free-text comments as they wish. The 23 questions cover 5 categories, including Usefulness, Ease of Use, Ease of Learning, Satisfaction and Privacy \& Data Sharing. A summary of the survey is shown in Figure 12.

Students generally felt positively about all aspects, and were particularly appreciative of the Ease of Use, Ease of Learning and Privacy \& Data Sharing dimensions. Additionally, students found the content provided valuable, given its being optional for the class. Despite the fact that there were various opinions on interface features, such as sorting and comparing, the overall attitude toward the system Usefulness was positive. The survey results support the design of the interface in terms of content organization. Students' positive responses also reinforce the objective system usage data. We also found that some of the students expressed their appreciation explicitly other than through ratings. Some of them wished the tools were offered for other courses. Some even suggested the alignment of the content for exams or usage for participation or credits.

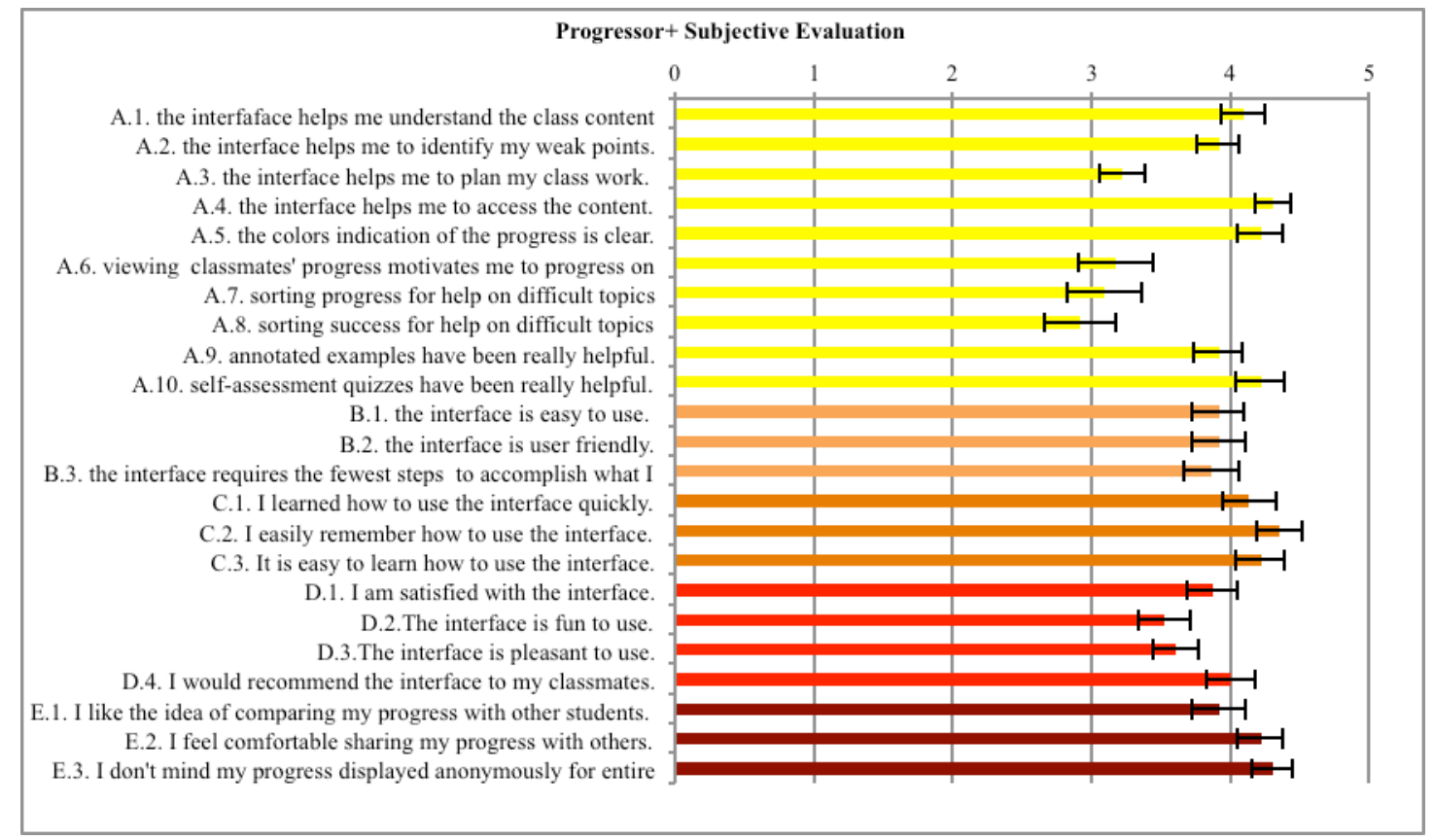

Figure 12. Summary of the subjective evaluation for each itemized survey question.

\section{Summary And Discussion}

To explore the value of open social student modeling, we developed several systems and conducted classroom studies to evaluate hypotheses and overall effectiveness. We learned from our experiences and improved the design for each implementation.

\subsection{Results Summary}

The classroom evaluation of our approach demonstrated that we achieved our main goal helping students to navigate a rich collection of learning resources. Providing navigation support through open social student modeling visualizations helped students to locate the most relevant content and achieve a significantly higher programming problem-solving success rate. In addition, incorporating a mixed collection of content in the OSSM visualizations effectively led the students to work at the right level of questions. Both stronger and weaker students worked with the appropriate levels of questions for their readiness, which yielded a consistent 
performance across all three levels of complexities. Additionally, providing a more realistic content collection on the navigation supported open social student modeling visualizations resulted in uniform performance for the group. The classroom study revealed a clear pattern of social guidance, where the stronger students left traces for weaker ones to follow. This effect was much more noticeable, especially for the Complex problems.

The analysis of our approach confirms that students spent more time on the system, attempted more self-assessment quizzes, and explored more annotated examples. They achieved a higher diversity in attempting the self-assessment questions and exploring the annotated examples. Students were motivated to do more work. They were engaged with the system; they spent about 5 hours for each collection. Moreover, they successfully achieved better learning results. Students obtained significantly higher knowledge gains than students without the support of the systems.

The subjective evaluation results showed that student generally felt positively about all aspects of the tool, particularly in terms of Ease of Use, Ease of Learning and Privacy \& Data Sharing. Additionally, students found the content provided was valuable. Despite the fact that there were various opinions on interface features, such as sorting and comparing, the overall attitude toward the system Usefulness was positive. These survey results confirm the design of the interface in terms of content organization. The students' positive responses also complement the objective system usage data.

\subsection{Contribution to the education field}

In this paper, we presented a series of innovative open social student modeling interfaces to support the learning of online programming language. We summarized the lessons learned from the design and classroom studies. We observed the impact of each design. The first contribution of this project is combining the ideas of adaptive navigational support and social visualization by using an open social student modeling interface. The combined approach lowers the modeling complexity for knowledge-based personalization and increases the precision of social navigation support among the increasingly large and diverse number educational resources. This approach decreases the threshold for semantic-enriched online education. It also brings online education closer to the modern classroom. In addition, the approach has been proven to guide students effectively to the right content at the right time. It could be one of the pioneer works in the open social student modeling realm.

Second, this work summarized the design principles for personalized guidance using open social student modeling visualization based on a series of pre-studies.

Third, this work established a scalable framework based on the design principles. The implementation, Progressor ${ }^{+}$, was evaluated in this study. This framework allows extending the content collections to simulate a more realistic online learning environment. In addition to eLearning, the classroom study also demonstrated that the tool can also be used as a complementary tool for real classrooms.

Forth, the underlying theories for adaptive navigational support and social visualization actually complement each other when brought together. According to learners' choices and beliefs about self-testing studies, students are generally overconfident about their memories and underestimate the amount they will learn by studying (Kornell \& Son, 2009). The overconfidence of understanding is more severe among less advanced learners \{Falchikov \& Boud, 1989), who need most to be improved (Falchikov \& Goldfinch, 2000). Therefore, this work unveiled the social comparison mechanism by providing comparative interfaces and demonstrating the stronger and weaker students' performances through quantitative analyses with this approach.

\subsection{Limitations \& future work}

All of the systems discussed were provided as supplemental tools for the same course. While we attempted to provide as realistic a scenario as possible by incorporating diverse learning objects for the learning environment, within the non-controlled classroom context students are still able to learn from the subject in many different ways. (i.e., having hands-on 
experiences in coding plays a very important role in the programming language learning context. In our curriculum, students claim to benefit the most from the laboratory sessions.) The system used is just one of the factors that contributed to the learning. The content collections used in this work did not cover all the knowledge taught in the programming course. However, we took into account the semantics questions when measuring the students' learning.

The first open social student modeling interface was introduced in the spring semester of 2010, while the latest system, Progressor ${ }^{+}$, was introduced in the spring semester of 2012. Because social technology is rapidly evolving, students could potentially have been exposed to mass social media within these two years and gradually become more comfortable with using social tools. Our study is not able to account for this phenomenon.

In addition, we recognize that current design supports implicit guidance, while the adaptive navigation support provides the personalized progress guidance and "wisdom of the crowd" leads the learning paths. In the future, we plan to enhance the explicit guidance, for example, by providing as recommendations. Using information about peers' prior success may allow us to recommended suitable topics to students where they have just failed. While the explicit recommendations in the user model visualization suggest more proactive personalized guidance, we will be facing the challenge of implementing this personalization without decreasing users' interest on making comparisons with their peers. However, we think that such issues can be addressed by enhancing the visualization, for example, by using different transparency levels to mark recommended and non-recommended topics.

\section{Reference}

Ahn, J. W., Brusilovsky, P., Grady, J., He, D., \& Syn, S. Y. (2007). Open user profiles for adaptive news systems: help or harm?. In Proceedings of the 16th international conference on World Wide Web (pp. 11-20). ACM.

Azevedo, R., Guthrie, J. T., \& Seibert, D. (2004). The role of self-regulated learning in fostering students' conceptual understanding of complex systems with hypermedia. Journal of Educational Computing Research, 30(1), 87-111.

Bakalov, F., König-Ries, B., Nauerz, A., \& Welsch, M. (2010). IntrospectiveViews: An interface for scrutinizing semantic user models. In User Modeling, Adaptation, and Personalization (pp. 219-230). Springer Berlin Heidelberg.

Barolli, L., Koyama, A., Durresi, A., \& De Marco, G. (2006). A web-based e-learning system for increasing study efficiency by stimulating learner's motivation. Information Systems Frontiers, 8(4), 297-306.

Brusilovsky, P. (2007). Adaptive navigation support. In P. Brusilovsky, A. Kobsa \& W. Neidl (Eds.), The Adaptive Web: Methods and Strategies of Web Personalization (Vol. 4321, pp. 263-290). Berlin Heidelberg New York: Springer-Verlag.

Brusilovsky, P., Chavan, G., \& Farzan, R. (2004, August 23-26, 2004). Social adaptive navigation support for open corpus electronic textbooks. Paper presented at the Third International Conference on Adaptive Hypermedia and Adaptive WebBased Systems (AH'2004), Eindhoven, the Netherlands.

Brusilovsky, P., Hsiao, I. H., \& Folajimi, Y. (2011). QuizMap: open social student modeling and adaptive navigation support with TreeMaps. In Towards Ubiquitous Learning (pp. 71-82). Springer Berlin Heidelberg.

Brusilovsky, P., Sosnovsky, S., \& Shcherbinina, O. (2004, November 1-5, 2004). QuizGuide: Increasing the Educational Value of Individualized Self-Assessment Quizzes with Adaptive Navigation Support. Paper presented at the World Conference on E- Learning, E-Learn 2004, Washington, DC, USA.

Brusilovsky, P., Sosnovsky, S., \& Yudelson, M. (2009). Addictive links: The motivational value of adaptive link annotation. New Review of Hypermedia and 
Multimedia, 15(1), 97-118.

Bull, S. (2004). Supporting learning with open learner models. Paper presented at the 4th Hellenic Conference on Information and Communication Technologies in Education, Athens, Greece.

Bull, S., \& Britland, M. (2007). Group Interaction Prompted by a Simple Assessed Open Learner Model that can be Optionally Released to Peers. Paper presented at the Workshop on Personalization in E-learning Environments at Individual and Group Level at the 11th International Conference on User Modeling, UM 2007, Corfu, Greece.

Bull, S., \& Kay, J. (2007). Student Models that Invite the Learner In: The SMILI:() Open Learner Modelling Framework. International Journal of Artificial Intelligence in Education, 17(Volume 17, Number 2 / 2007), 89-120.

Bull, S., \& Kay, J. (2013). Open learner models as drivers for metacognitive processes. In International handbook of metacognition and learning technologies (pp. 349-365). Springer New York.

Buunk, A. P., \& Gibbons, F. X. (2007). Social comparison: The end of a theory and the emergence of a field. Organizational Behavior and Human Decision Processes, 102(1), 3-21.

Chen, Z.-H., Chou, C.-Y., Deng, Y.-C., \& Chan, T.-W. (2007). Active Open Learner Models as Animal Companions: Motivating Children to Learn through Interacting with My- Pet and Our-Pet. International Journal of Artificial Intelligence in Education, 17(Volume 17, Number 2 / 2007), 145-167.

Cialdini, R. B., Wosinska, W., Barrett, D. W., Butner, J., \& Gornik-Durose, M. (1999). Compliance with a request in two cultures: The differential influence of social proof and commitment/consistency on collectivists and individualists. Personality and Social Psychology Bulletin, 25(10), 1242-1253.

Corbett, A. T., Anderson, J. R., \& O'Brien, A. T. (1995). Student modeling in the ACT programming tutor. Cognitively diagnostic assessment, 19-41.

Darnon, C., Dompnier, B., Gilliéron, O., \& Butera, F. (2010). The interplay of mastery and performance goals in social comparison: A multiple-goal perspective. Journal of Educational Psychology, 102(1), 212.

Dieberger, A. (1997). Supporting social navigation on the World Wide Web.

International Journal of Human-Computer Interaction, 46, 805-825.

Dieberger, A., Dourish, P., Höök, K., Resnick, P., \& Wexelblat, A. (2000). Social navigation: Techniques for building more usable systems. interactions, 7(6), 36-45. Dijkstra, P., Kuyper, H., Werf, G. v. d., Buunk, A. P., \& Zee, Y. G. v. d. (2008).

Social Comparison in the Classroom: A Review. Review Of Educational

Research, 78(4).

Dimitrova, V. (2003). STyLE-OLM: Interactive open learner modelling. International Journal of Artificial Intelligence in Education, 13(1), 35-78.

Falchikov, N., \& Boud, D. (1989). Student Self-Assessment in Higher Education: A Meta- Analysis. REVIEW OF EDUCATIONAL RESEARCH, 59(4), 395-430. Falchikov, N., \& Goldfinch, J. (2000). Student Peer Assessment in Higher Education: A Meta-Analysis Comparing Peer and Teacher Marks. REVIEW OF EDUCATIONAL RESEARCH, 70(3), 287-322.

Farzan, R., \& Brusilovsky, P. (2008). AnnotatEd: A social navigation and annotation 
service for web-based educational resources. New Review in Hypermedia and Multimedia, 14(1), 3-32.

Festinger, L. (1954). A theory of social comparison processes. Human Relations, 7, 117-140. Freeman, L. C. (2000). Visualizing Social Networks. Journal of Social Structure, 1. Gershon, N., \& Page, W. (2001). What storytelling can do for information visualization. Commun. ACM, 44(8), 31-37.

Hsiao, I.-H., Bakalov, F., Brusilovsky, P., \& König-Ries, B. (2011). Open Social Student Modeling: Visualizing Student Models with Parallel IntrospectiveViews. Paper presented at the 19th International Conference on User Modeling, Adaptation and Personalization (UMAP 2011), Girona, Spain.

Hsiao, I. H., \& Brusilovsky, P. (2012). Motivational social visualizations for personalized E-learning. In 21st Century Learning for 21st Century Skills (pp. 153-165). Springer Berlin Heidelberg.

Hsiao, I.-H., Brusilovsky, P., \& Sosnovsky, S. (2008). Web- based Parameterized Questions for Object-Oriented Programming. Paper presented at the World Conference on E-Learning, E-Learn 2008, Las Vegas, USA.

Hsiao, I.-H., Sosnovsky, S., \& Brusilovsky, P. (2010). Guiding students to the right questions: adaptive navigation support in an E-Learning system for Java programming. Journal of Computer Assisted Learning, 26(4), 270-283.

Huguet, P., Dumas, F., Monteil, J. M., \& Genestoux, N. (2001). Social comparison choices in the classroom: Further evidence for students' upward comparison tendency and its beneficial impact on performance. European journal of social psychology, 31(5), 557-578.

Kaplan, A., \& Maehr, M. L. (2007). The contributions and prospects of goal orientation theory. Educational Psychology Review, 19(2), 141-184.

Kay, J. (1997). Learner know thyself: Student models to give learner control and responsibility. Paper presented at the ICCE97, International Conference on Computers in Education, Malasia, Kuching, Sarawak.

Kay., J. (2008). Lifelong Learner Modeling for Lifelong Personalized Pervasive Learning. IEEE Transaction on Learning Technologies, 1(4), 215-228.

Kornell, N., \& Son, L. K. (2009). Learners' choices and beliefs about self-testing. [doi:10.1080/09658210902832915]. Memory, 17(5), 493-501.

Kurhila, J., Miettinen, M., Nokelainen, P., \& Tirri, H. (2006). Educo- A Collaborative Learning Environment Based on Social Navigation Adaptive Hypermedia and Adaptive Web-Based Systems. In P. De Bra, P. Brusilovsky \& R. Conejo (Eds.), (Vol. 2347, pp. 242-252): Springer Berlin / Heidelberg.

Light, P., Littleton, K., Bale, S., Joiner, R., \& Messer, D. (2000). Gender and social comparison effects in computer-based problem solving. Learning and Instruction, 10(6), 483-496.

Linton, F., Joy, D., Schaefer, H. P., \& Charron, A. (2000). OWL: A recommender system for organization-wide learning. Educational Technology \& Society, 3(1), 62-76.

Mabbott, A., \& Bull, S. (2004). Alternative views on knowledge: Presentation of open learner models. In Intelligent tutoring systems (pp. 689-698). Springer Berlin Heidelberg.

Mazza, R., \& Dimitrova, V. (2007). CourseVis: A graphical student monitoring tool for supporting instructors in web-based distance courses. [doi: 10.1016/j.ijhcs.2006.08.008]. International Journal of Human-Computer Studies, 
65(2), $125-139$.

Méndez, J. A., Lorenzo, C., Acosta, L., Torres, S., \& González, E. (2006). A webbased tool for control engineering teaching. [10.1002/cae.20080]. Computer Applications in Engineering Education, 14(3), 178-187.

Mitrovic, A., \& Martin, B. (2007). Evaluating the Effect of Open Student Models on Self- Assessment. International Journal of Artificial Intelligence in Education, 17(2), $121-144$.

Pintrich, P. R., \& De Groot, E. V. (1990). Motivational and self-regulated learning components of classroom academic performance. Journal of educational psychology, 82(1), 33 .

Rao, R., \& Card, S. K. (1994). The table lens: merging graphical and symbolic representations in an interactive focus + context visualization for tabular information. Paper presented at the Proceedings of the SIGCHI conference on Human factors in computing systems: celebrating interdependence.

Shneiderman, B. (1996). The eyes have it: A task by data type taxonomy for information visualizations. Paper presented at the Symposium on Visual Languages, Washington D.C.

Shneiderman,B. (2004). Treemaps for Space Constrained Visualization of Hierarchies: an historical summary of Treemap research and applications. from http://www.cs.umd.edu/hcil/treemaps/

Triantafillou, E., Pomportsis, A., Demetriadis, S., \& Georgiadou, E. (2004). The value of adaptivity based on cognitive style: an empirical study. British Journal of Educational Technology, 35(1), 95-106.

Tufte, E. R. (1990). Envisioning Information. Cheshire, CT: Graphics Press.

Vassileva, J. (2008). Toward Social Learning Enviroments. IEEE Transaction on Learning Technologies, 1(4), 199-214.

Vassileva, J., \& Sun, L. (2007). Using Community Visualization to Stimulate Participation in Online Communities. e-Service Journal. Special Issue on Groupware, 6(1), 3-40.

Vassileva, J., \& Sun, L. (2008). Evolving a Social Visualization Design Aimed at Increasing Participation in a Class-Based Online Community. International Journa of Cooperative Information Systems (IJCIS), 17(4), 443-466.

Wattenberg, M. (1999). Visualizing the stock market. Paper presented at the CHI '99 extended abstracts on Human factors in computing systems.

Weber, G., \& Brusilovsky, P. (2001). ELM-ART: An adaptive versatile system for Web- based instruction. International Journal of Artificial Intelligence in Education, 12(4), 351-384.

Zapata-Rivera, J.-D., \& Greer, J. E. (2000). Inspecting and Visualizing Distributed Bayesian Student Models. Paper presented at the 5th International conference Intelligent Tutoring Systtems.

Zimmerman, B. J. (1990). Self-Regulated Learning and Academic Achievement: An Overview. [doi: 10.1207/s15326985ep2501_2]. Educational Psychologist, 25(1), 317. 